\title{
Lysosomal degradation of newly formed insulin granules contributes to $\beta$ cell failure in diabetes
}

\author{
Adrien Pasquier 1,2,3,4, Kevin Vivot 1,2,3,4, Eric Erbs ${ }^{1,2,3,4}$, Coralie Spiegelhalter 1,2,3,4, Zhirong Zhang 1,2,3,4, \\ Victor Aubert1,2,3,4, Zengzhen Liu1,2,3,4, Meryem Senkara5 ${ }^{5}$, Elisa Maillard ${ }^{4,6}$, Michel Pinget ${ }^{4,6}$, Julie Kerr-Conte , \\ François Pattou (iD ${ }^{7}$, Gilbert Marciniak ${ }^{8}$, Axel Ganzhorn ${ }^{8}$, Paolo Ronchi ${ }^{9}$, Nicole L. Schieber (iD ${ }^{9}$, \\ Yannick Schwab (1) 9,10, Paul Saftig ${ }^{5}$, Alexander Goginashvili (10) 1,2,3,4,12,13 \& Romeo Ricci, 1,2,3,4,11,13
}

Compromised function of insulin-secreting pancreatic $\beta$ cells is central to the development and progression of Type 2 Diabetes (T2D). However, the mechanisms underlying $\beta$ cell failure remain incompletely understood. Here, we report that metabolic stress markedly enhances macroautophagy-independent lysosomal degradation of nascent insulin granules. In different model systems of diabetes including of human origin, stress-induced nascent granule degradation (SINGD) contributes to loss of insulin along with mammalian/ mechanistic Target of Rapamycin (mTOR)-dependent suppression of macroautophagy. Expression of Protein Kinase D (PKD), a negative regulator of SINGD, is reduced in diabetic $\beta$ cells. Pharmacological activation of PKD counters SINGD and delays the onset of T2D. Conversely, inhibition of PKD exacerbates SINGD, mitigates insulin secretion and accelerates diabetes. Finally, reduced levels of lysosomal tetraspanin CD63 prevent SINGD, leading to increased insulin secretion. Overall, our findings implicate aberrant SINGD in the pathogenesis of diabetes and suggest new therapeutic strategies to prevent $\beta$ cell failure.

\footnotetext{
${ }^{1}$ Institut de Génétique et de Biologie Moléculaire et Cellulaire, 67404 IIIkirch, France. ${ }^{2}$ Centre National de la Recherche Scientifique, UMR7104, 67404 IIlkirch, France. ${ }^{3}$ Institut National de la Santé et de la Recherche Médicale, U964, 67404 Illkirch, France. ${ }^{4}$ Université de Strasbourg, 67081 Strasbourg, France. ${ }^{5}$ Biochemisches Institut, Christian-Albrechts Universität Kiel, D-24118 Kiel, Germany. ${ }^{6}$ Centre Européen d'Etude du Diabète (CEED), DIATHEC EA 7294, F-67000 Strasbourg, France. ${ }^{7}$ Institut National de la Santé et de la Recherche Médicale U1190, EGID, CHU Lille, University of Lille, 59000 Lille, France. 8 Open Innovation Access Platform, Sanofi-Aventis R\&D, 67080 Strasbourg, France. ${ }^{9}$ European Molecular Biology Laboratory, Electron Microscopy Core Facility, 69117 Heidelberg, Germany. ${ }^{10}$ European Molecular Biology Laboratory, Cell Biology and Biophysics Unit, 69117 Heidelberg, Germany. ${ }^{11}$ Laboratoire de Biochimie et de Biologie Moléculaire, Nouvel Hôpital Civil, 67091 Strasbourg, France. ${ }^{12}$ Present address: Ludwig Institute for Cancer Research; Department of Cellular and Molecular Medicine, University of California San Diego, La Jolla, CA 92093, USA. ${ }^{13}$ These authors jointly supervised this work: Alexander Goginashvili, Romeo Ricci. Correspondence and requests for materials should be addressed to A.G. (email: agoginashvili@ucsd.edu) or to R.R. (email: romeo.ricci@igbmc.fr)
} 
C ells employ unique evolutionary programmed strategies to cope with changes in nutrient availability. In complex organisms, these cellular responses are coordinated through hormonal cues with the aim to maintain whole-body metabolic homeostasis. The pancreatic $\beta$ cell harbors an exemplary nutrient sensing machinery coupled to insulin secretion, which controls glucose homeostasis in normal conditions and is disturbed in diabetes.

Macroautophagy is a major mechanism used by the cells to remove damaged organelles and unused or aggregated proteins. In addition, it helps maintaining cellular homeostasis and is activated in response to nutrient shortage ${ }^{1-3}$. Macroautophagy involves formation of cargo-containing autophagosomes, which is controlled by a complex molecular machinery consisting of the autophagy-related (ATG) proteins ${ }^{4}$. Autophagosomes subsequently fuse with lysosomes to generate autolysosomes in which degradation occurs.

Several studies have demonstrated that macroautophagy is crucial to maintain $\beta$ cell function in response to cellular stress linked to T2D. Evidence for the protective role of macroautophagy has been provided in high-fat diet-induced obesity $^{5,6}$, in Endoplasmic Reticulum (ER) stressinduced diabetes 7,8 , in human islet amyloid polypeptideinduced diabetes ${ }^{9-12}$ as well as in lipo- and glucotoxicity models ${ }^{13-16}$. Importantly, both enhanced $5,7,15,17$ and reduced macroautophagy $13,16,18$ have been reported in metabolically challenged $\beta$ cells. These seemingly controversial observations can be explained by the fact that macroautophagy levels are high and protective in compensated $\beta$ cells in pre-diabetes, while they decrease at later stages, which contributes to $\beta$ cell failure. In fact, depending on the nature and duration of metabolic challenges, levels of macroautophagy varied accordingly ${ }^{15,19}$. Finally, consistent with perturbed autophagic flux, accumulation of autophagosomes has been observed in $\beta$ cells of islets isolated from T2D patients ${ }^{20,21}$.

Pancreatic $\beta$ cells dedicate up to $50 \%$ of their protein biosynthesis to insulin production ${ }^{22}$, requiring a tight control of insulin granule homeostasis. While mechanisms governing insulin granule biogenesis and exocytosis in response to nutrients were widely investigated ${ }^{23,24}$, little is known about how nutrients impact on insulin granule turnover. Targeting of insulin granules to the lysosomes has been reported to occur in autophagosomedependent and -independent manner ${ }^{25-31}$.

Recently we have described a macroautophagy-independent mechanism in pancreatic $\beta$ cells as an immediate response for them to adapt to nutrient depletion ${ }^{32}$. We have demonstrated that nutrient-deprived $\beta$ cells deliver newly synthesized secretory granules (SGs) to lysosomes in the Golgi area, where degradation of their cargo (proinsulin and insulin) provides necessary nutrients leading to mTOR-mediated suppression of macroautophagy. Starvation-induced nascent granule degradation allows $\beta$ cells to cope with shortage of nutrients and to prevent unwanted insulin release. As uncontrolled lysosomal degradation of insulin granules may provoke loss of insulin and macroautophagy dysfunction, both of which occur in diabetic $\beta$ cells, we wondered whether aberrant activation of this pathway might be implicated in $\beta$ cell failure in T2D.

To test this hypothesis, we now focus on several models, including metabolically stressed $\beta$ cell lines, human and mouse pancreatic islets as well as diabetic mice. In all of these, we find markedly increased lysosomal degradation of nascent insulin granules, which results in depletion of insulin and mTORdependent suppression of macroautophagy. As this pathway is not restricted to conditions of nutrient deprivation, we now refer to it as stress-induced nascent granule degradation (SINGD, pronounced 'sIndi).
Furthermore, we provide evidence in vivo that pharmacological inhibition of SINGD delays onset of T2D, while activation of SINGD accelerates its progression. Finally, we find that the tetraspanin CD63 determines the destination of nascent insulin granules towards degradation versus secretion. Collectively, our findings delineate a mechanism that links impaired signaling at the Golgi to $\beta$ cell failure in T2D.

\section{Results}

Metabolic stress enhances SINGD. We first investigated SINGD in $\beta$ cells upon chronic exposure to elevated levels of Glucose (Glc) and Palmitate (Pal), i.e., glucolipotoxic conditions ${ }^{33}$. We treated rat insulinoma-derived $\beta$ cells (INS1 cells) with $33.3 \mathrm{mM}$ Glc and $0.4 \mathrm{mM} \mathrm{Pal}(\mathrm{Glc} / \mathrm{Pal})$ or $11 \mathrm{mM}$ Glc (control growing culture) for $20 \mathrm{~h}$. We observed that $\mathrm{Glc} / \mathrm{Pal}$ led to a drastic decrease in insulin-positive SGs at the plasma membrane in comparison to control conditions. Importantly, Glc/Pal-treated cells also accumulated SGs in the Giantin-positive Golgi area (Fig. 1a, Supplementary Fig. 1a) prompting us to further characterize the latter. Immunofluorescence of proinsulin/insulin ((pro)insulin) and CD63 as markers of SGs and late endosomes/ lysosomes respectively, revealed abundant large CD63 puncta, colocalizing with (pro)insulin in the Golgi area in Glc/Pal-treated INS1 in comparison to control cells (Fig. 1b). Accordingly, increased co-localization of (pro)insulin with another lysosomal marker Lysosomal-Associated Membrane Protein 2 (LAMP2) was found in the Golgi area upon Glc/Pal as compared to control treatment (Supplementary Fig. 1b). Collectively, these findings hint at potential induction of SINGD upon glucolipotoxic conditions.

To address the dynamics of SINGD in metabolically challenged cells, we generated INS1 cells endogenously expressing the SG marker Phogrin tagged with Green Fluorescent Protein (GFP) and the lysosomal marker CD63 tagged with Discosoma sp. Red fluorescent protein (DsRed) (hereafter referred to as INS1 PGCD cells). Using CRISPR-Cas9 gene editing ${ }^{34}$, we added sequences coding corresponding fluorescent proteins to the $3^{\prime}$ end of the last exon of PTPRN2 (or Phogrin) and CD63, respectively (Supplementary Fig. 2a-j). INS1 ${ }^{P G C D}$ cells contain a homozygous insertion of GFP in the Phogrin locus, and a heterozygous insertion of DsRed in the CD63 locus, confirmed by Sanger sequencing and PCR analysis (Supplementary Fig. 2b, d, g, h). The expression of Phogrin-GFPendo was corroborated by immunobloting (Supplementary Fig. 2c) and cellular localization of Phogrin-GFPendo and CD63-DsRed ${ }^{\text {endo }}$ was confirmed by immunofluorescence and correlative light and electron microscopy (CLEM) (Supplementary Fig. 2e, i, j). In line with SINGD triggered by starvation ${ }^{32}$, INS1 ${ }^{\text {PGCD }}$ cells demonstrated increased Phogrin/CD63 co-localization upon nutrient deprivation as compared to control conditions (Supplementary Fig. 3a). Accordingly, inhibition of Protein Kinase D (PKD), a major negative regulator of $\mathrm{SINGD}^{32}$, using the selective $\mathrm{PKD}$ inhibitor CID755673 35 also resulted in increased Phogrin/CD63 colocalization (Supplementary Fig. 3b). Time-lapse microscopy of INS1 ${ }^{\text {PGCD }}$ cells treated with Glc/Pal revealed a marked increase in Phogrin/CD63 co-localization starting already after $6 \mathrm{~h}$ of treatment (Fig. 1c). Interestingly, INS1PGCD cells treated separately with Glc or Pal also demonstrated increased Phogrin/CD63 co-localization indicating that various inducers of metabolic stress are capable to trigger SINGD (Supplementary Fig. 3c).

We next tested whether SINGD induced by Glc/Pal occurred via macroautophagy. To this end, we followed SG markers, (pro) insulin and Phogrin, in INS1 1 LC3B-GFPendo cells endogenously expressing $\mathrm{LC} 3 \mathrm{~B}$ tagged with $\mathrm{GFP}^{32}$, a marker for autophagic 
a

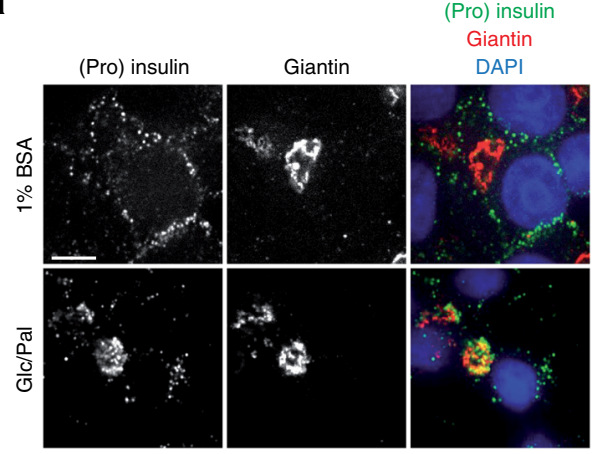

C
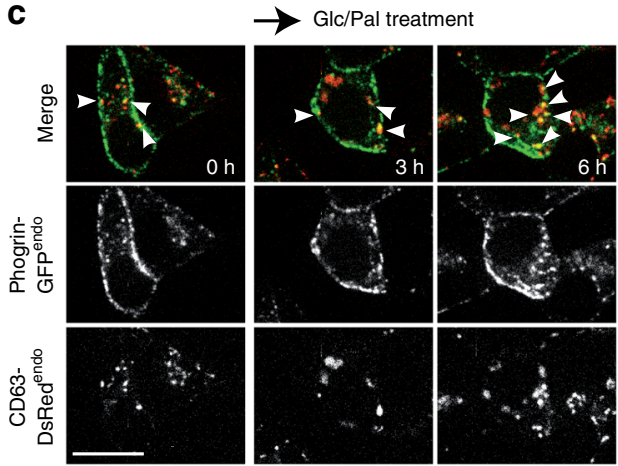

b

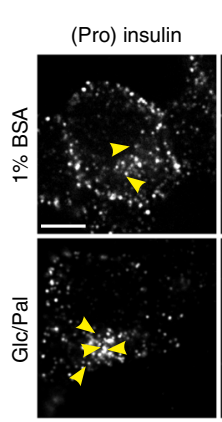

$\mathrm{CD} 63$

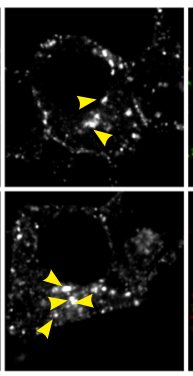

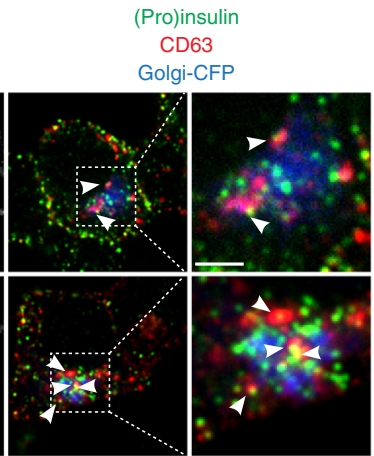

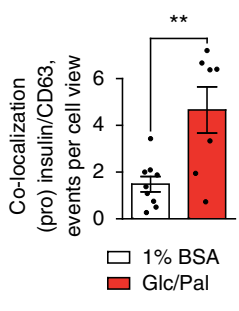

INS1 cells endogenously expressing Phogrin-GFP and CD63-DsRed (INS1 ${ }^{\text {PGCD }}$

e

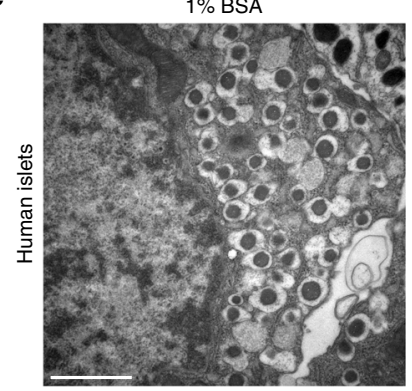

Glc/Pal 72 h

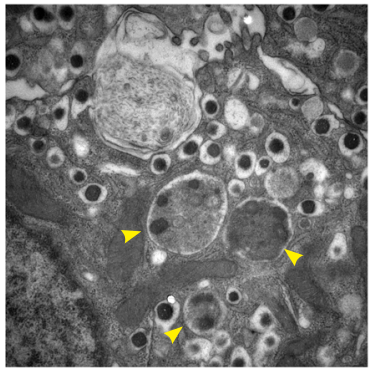

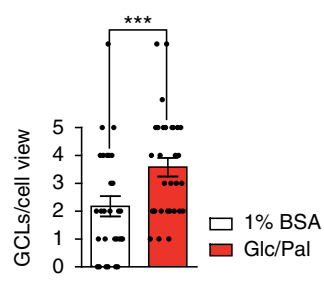

d

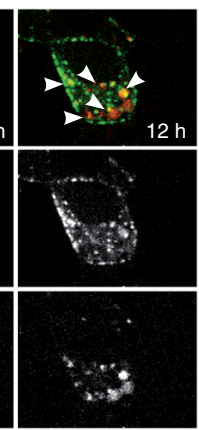

CD63-DsRed $d^{\text {endo }}$

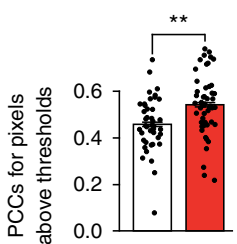

ㅁ $1 \% \mathrm{BSA}$ $\square \mathrm{Glc} / \mathrm{Pal}$

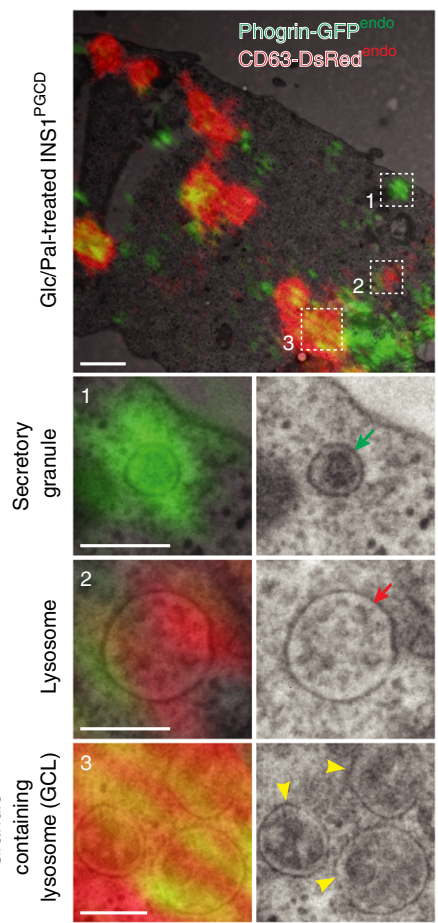

Fig. 1 Targeting of secretory granules to lysosomes in response to glucolipotoxicity in $\beta$ cells. a Immunofluorescence (IF) of insulin + proinsulin ("(pro) insulin") (green) and giantin (red) in INS1 cells treated with control (1\% BSA, $11 \mathrm{mM}$ glucose, "1\% BSA") or glucolipotoxic (1\% BSA, $33.3 \mathrm{mM}$ Glucose and $0.4 \mathrm{mM}$ Palmitate, "Glc/Pal") media for $20 \mathrm{~h}$. Nuclei were stained with DAPI. Scale bar, $5 \mu \mathrm{m}$. b IF of (pro)insulin (green) and CD63 (red) in INS1 cells treated with $1 \%$ BSA or Glc/Pal media for $20 \mathrm{~h}$. Golgi-CFP was used to visualize the Golgi apparatus. Arrowheads point to (pro)insulin/CD63 co-localization. Scale bar, $5 \mu \mathrm{m}$; for insets: $2 \mu \mathrm{m}$. Quantification of co-localization events between (pro)insulin and CD63 per cell view. Fields from three independent experiments, $N_{1 \% \mathrm{BSA}}=9 ; N_{\mathrm{Gl} / \mathrm{Pal}}=11, n$ cells: 82 and 64, respectively; ${ }^{\star \star} P<0.01$, two-tailed $t$-test. c Imaging of INS1PGCD cells endogenously expressing Phogrin-GFP (Phogrin-GFPendo) and CD63-DsRed (CD63-DsRedendo) treated with Glc/Pal for indicated time periods. Arrowheads point to Phogrin-GFPendo/CD63DsRed ${ }^{\text {endo }}$ co-localization. Scale bar, $20 \mu \mathrm{m}$. Quantification of co-localization between CD63-DsRedendo and Phogrin-GFPendo using Pearson's correlation. $N_{1 \% B S A}=42 ; N_{G l c / \text { Pal }}=50$ from three independent experiments; ${ }^{\star \star} P<0.01$, two-tailed $t$-test. $\mathbf{d}$ Correlative light and electron microscopy analysis of INS1PGCD cells treated with $\mathrm{Glc} / \mathrm{Pal}$ for $20 \mathrm{~h}$. Yellow arrowheads point to granule-containing lysosomes (GCLs). Green and red arrows point to a secretory granule and a lysosome respectively. Scale bar, $1 \mu \mathrm{m}$; for insets: $0.3 \mu \mathrm{m}$. e Electron microscopy analysis of $\beta$ cells in human islets treated with $1 \% \mathrm{BSA}$ or Glc/Pal media for $72 \mathrm{~h}$. Yellow arrowheads point to GCLs. Scale bar, $1 \mu \mathrm{m}$. Quantification of GCLs per cell view. $N_{1 \% B S A}=29 ; N_{\mathrm{Gl}} /$ Pal $=31$ from three independent experiments; ${ }^{\star \star \star} P<0.001$, two-tailed $t$-test. In $\mathbf{b}, \mathbf{c}$, e data are shown as mean $\pm S E M$, source data are provided as a Source Data file

intermediate compartments - autophagosomes ${ }^{36}$. While we observed occasional autophagosomes containing SGs in control-treated cells, the amount of SG-positive autophagosomes was almost abolished upon $\mathrm{Glc} / \mathrm{Pal}$ treatment, suggesting that SINGD does not rely on macroautophagy (Supplementary Fig. 4a, b).

We next monitored SINGD upon downregulation of the major canonical macroautophagy genes atg5 and beclin1 (becn1) that play a crucial role in early steps of the macroautophagy pathway by controlling the biogenesis of autophagosomes ${ }^{4}$. The lysosomal v-ATPase inhibitor Bafilomycin A1 (BafA1) counters lysosome activity and prevents the fusion of autophagosomes with lysosomes 37,38 , and thus is routinely used to measure autophagic flux. As expected, silencing of atg5 and beclin1 led to a drastic decrease in the number of LC3B-GFP-positive puncta in BafA1-treated Glc/Pal-treated INS1 ${ }^{\text {LC3B-GFPendo }}$ cells, indicating that early steps of macroautophagy were inhibited prior formation of autophagosomes (Supplementary Fig. 4c, d). However, in line with macroautophagy-independent SINGD ${ }^{32}$, silencing of atg5 and beclin1 did not lead to any decrease in Phogrin/CD63 co-localization upon Glc/Pal (Supplementary Fig. $4 \mathrm{e}-\mathrm{g})$. 
Importantly, BafA1 markedly increased the amount of LC3Bpositive/CD63-negative puncta in Glc/Pal-treated INS1 ${ }^{\text {PGCD }}$ cells, in line with accumulation of autophagosomes upon inhibition of fusion of autophagosomes with lysosomes (Supplementary Fig. 4h). If Glc/Pal-induced delivery of SGs to lysosomes occurred via autophagosomes, BafA1 treatment would lead to accumulation of SG-containing autophagosomes in the cytoplasm, preventing delivery to CD63-positive lysosomes. However, we observed the opposite: the LC3B-positive/CD63-negative autophagosomes did not contain SGs; and the size and amount of co-localized Phogrin/CD63 signals was further increased upon BafA1, overall corroborating macroautophagy-independent SINGD (Supplementary Fig. 4i, j).

We next used correlative light and electron microscopy (CLEM) to follow SINGD at the ultrastructural level. First, CLEM of Glc/Pal-treated INS1 ${ }^{\text {PGCD }}$ cells confirmed large CD63and Phogrin-positive granule-containing lysosomes (GCLs) (Fig. 1d and Supplementary Fig. 5a). Second, live-cell imaging followed by CLEM (live-CLEM) identified that GCLs were formed via direct fusion between SGs and lysosomes (Supplementary Fig. $5 \mathrm{~b}$ and Supplementary Movie 1).

Finally, $\beta$ cells of primary human islets, treated with Glc/Pal for $72 \mathrm{~h}$ contained abundant GCLs in the Golgi area, as revealed by quantitative Electron Microscopy (EM) analysis (Fig. 1f). Altogether, our data indicate that prolonged exposure of $\beta$ cells to $\mathrm{Glc} / \mathrm{Pal}$ diverts SGs from the secretory route to lysosomes in a macroautophagy-independent manner.
mTOR suppresses macroautophagy upon metabolic stress. Activation of mammalian/mechanistic Target of Rapamycin (mTOR) Complex 1 occurs at the lysosomal membrane in response to addition of amino acids ${ }^{39-41}$. We have recently shown that SINGD triggered by starvation was associated with increased recruitment of mTOR to GCLs, mTOR activation and suppression of macroautophagy via mTOR-mediated inhibitory phosphorylation of Unc-51-like kinase 1 (ULK1) ${ }^{32}$. We thus next asked whether nutrient stress imposed by $\mathrm{Glc} / \mathrm{Pal}$ treatment evoked similar effects. In fact, prolonged Glc/Pal treatment was shown to induce macroautophagy dysfunction in a mTOR-dependent manner ${ }^{13,16}$. We observed that $\mathrm{Glc} / \mathrm{Pal}$ recruited mTOR to CD63-positive lysosomes in INS1 cells (Fig. 2a, Supplementary Movie 2) and increased phospho-ULK1 (Fig. 2b). Moreover,

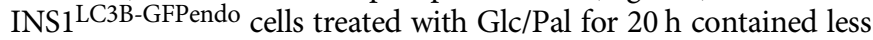
LC3B-GFP puncta as compared to control-treated cells (Fig. 2c). Accordingly, immunoblotting revealed a decrease in lipidated autophagosomal LC3B-II in Glc/Pal-treated INS1 cells and primary human islets as compared to control conditions. This difference was evident in the presence and absence of BafA1, indicating reduced autophagy flux (Fig. 2d, quantified in Supplementary Fig. 6). Consistent with mTOR-mediated suppression of macroautophagy in Glc/Pal-treated cells, the mTOR inhibitor rapamycin increased LC3B-GFP puncta in Glc/Pal-treated INS1 ${ }^{\text {LC3B-GFPendo }}$ cells (Fig. 2e). Altogether, these data indicate that lysosomal degradation of SGs is likely to contribute to mTOR-dependent suppression of macroautophagy in metabolically stressed $\beta$ cells.
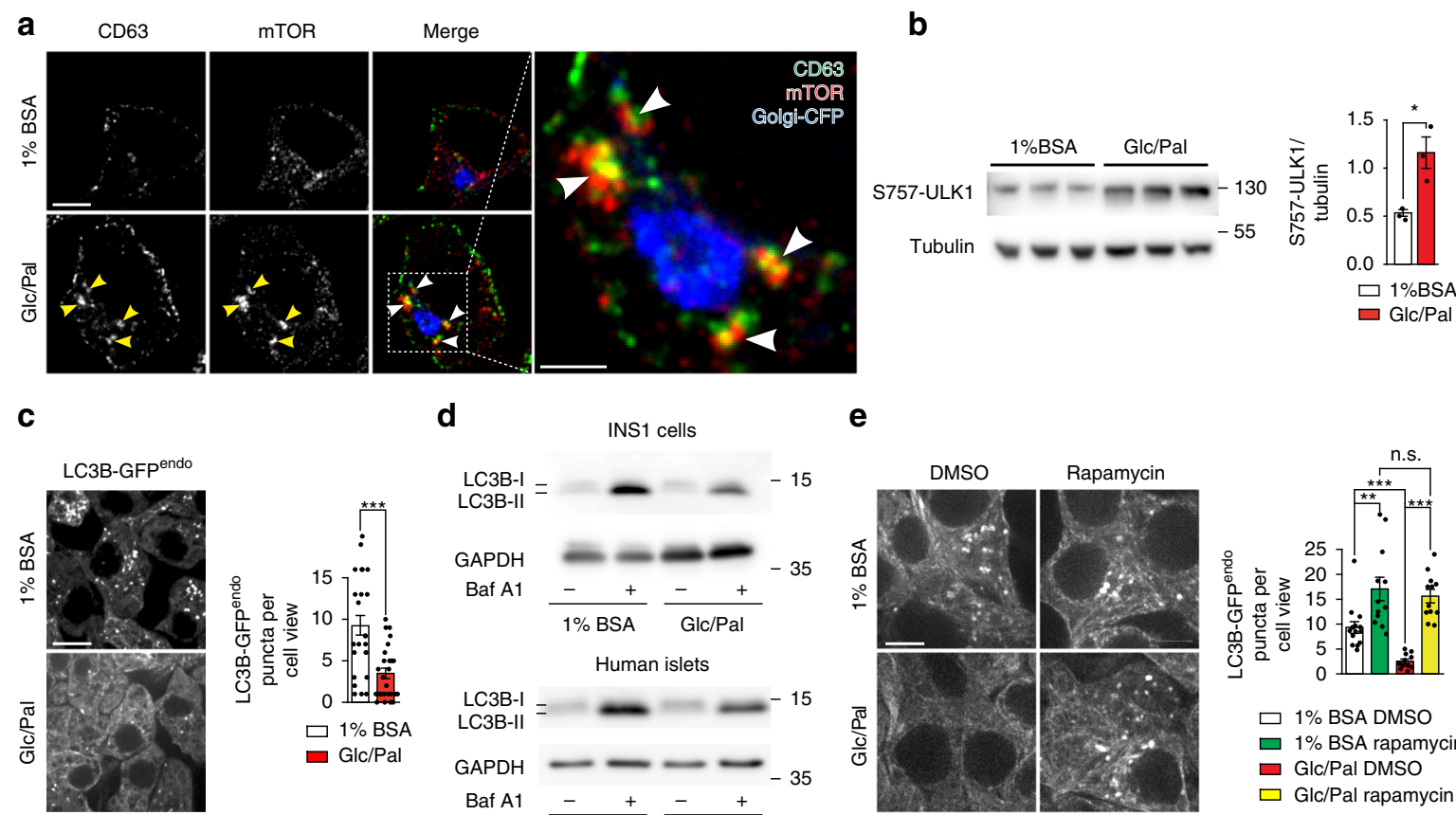

d
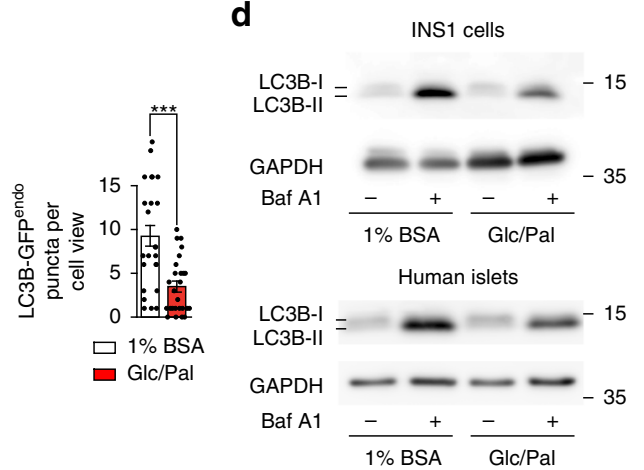

e
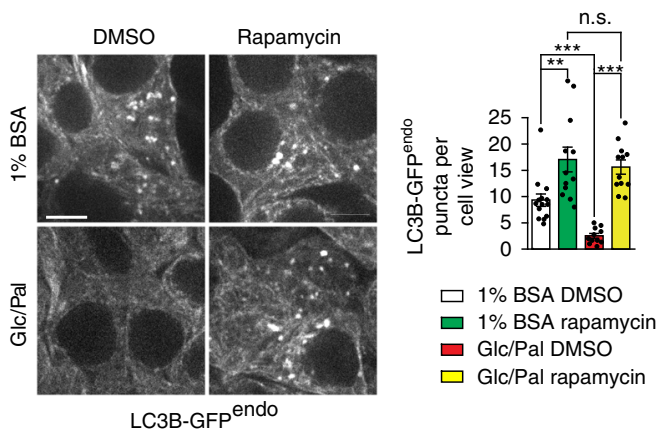

Fig. 2 Glucolipotoxicity leads to mTOR-dependent inhibition of macroautophagy in $\beta$ cells. a Immunofluorescence of CD63 (green) and mTOR (red) in INS1 cells treated with control (1\% BSA, $11 \mathrm{mM}$ glucose, "1\% BSA") or glucolipotoxic (1\% BSA, $33.3 \mathrm{mM}$ Glucose and $0.4 \mathrm{mM} \mathrm{Palmitate,} \mathrm{"Glc/Pal")} \mathrm{media}$ for $20 \mathrm{~h}$. Golgi-CFP was used to visualize the Golgi apparatus. Arrowheads point to large CD63/mTOR puncta. Scale bar, $5 \mu \mathrm{m}$; for inset: $2 \mu \mathrm{m}$. $3 \mathrm{D}$ reconstruction is shown in Supplementary Movie 2. b Immunoblot of S757-ULK1 using lysates of INS1 cells treated with $1 \%$ BSA or Glc/Pal media for $48 \mathrm{~h}$. Tubulin was used as a loading control. Quantification of S757-ULK1/Tubulin ratio. N=3 per group; ${ }^{*} P<0.05$, two-tailed $t$-test. c LC3B-GFPendo puncta in INS1 cells endogenously expressing LC3B-GFP (INS1LC3B-GFPendo) treated with 1\% BSA or Glc/Pal media for $40 \mathrm{~h}$. Scale bar, $10 \mu \mathrm{m}$. Quantification of the puncta per cell view, two independent experiments, $N_{1 \% B S A}=24 ; N_{\mathrm{Glc} / \text { Pal }}=26 ;{ }^{\star \star \star} P<0.001$, two-tailed $t$-test. $\mathbf{d}$ Immunoblot of LC3B using lysates of INS1 cells (top) or human islets (bottom) treated with $1 \% \mathrm{BSA}$ or Glc/Pal for $40 \mathrm{~h}$ in the presence or absence of bafilomycin A1 (BafA1), $10 \mathrm{nM}$ added for the last $1 \mathrm{~h}$ (INS1) or $2 \mathrm{~h}$ (islets) of incubation. GAPDH was used as a loading control. e LC3B-GFpendo puncta in INS1 LC3B-GFPendo cells treated for $40 \mathrm{~h}$ as indicated, DMSO or rapamycin $(100 \mathrm{nM})$ were added for the last $2 \mathrm{~h}$ of incubation. Scale bar, $5 \mu \mathrm{m}$. Quantification of puncta per cell view. Fields from three independent experiments, $N_{1 \% B S A}$ DMSO $=14 ; N_{1 \% B S A}$ Rapamycin $=12 ; N_{\text {Glc } / \text { Pal DMSO }}=12 ; N_{\text {Glc } / \text { Pal Rapamycin }}=12 ; n$ cells: $50,32,32,28$ respectively; ${ }^{\star \star} P<0.01$, ${ }^{\star \star \star} P<0.001$, n.s.: not significant, two-tailed $t$-test. In $\mathbf{b}, \mathbf{c}$, e data are shown as mean \pm SEM, source data are provided as a Source Data file 
a

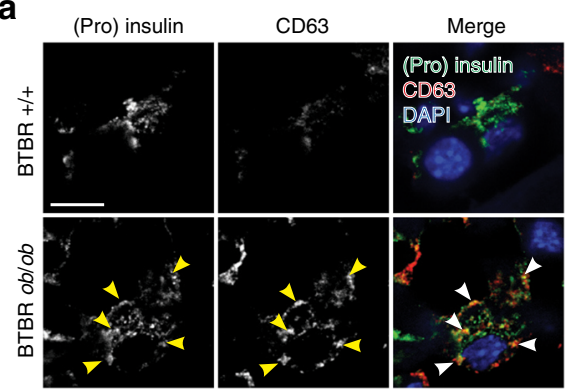

C b

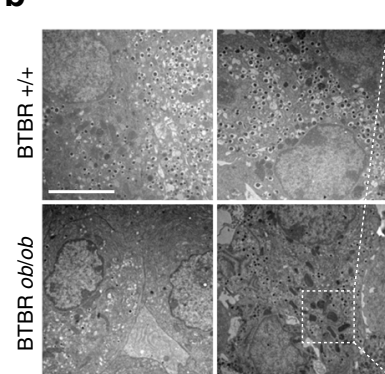

Golgi area with GCLs

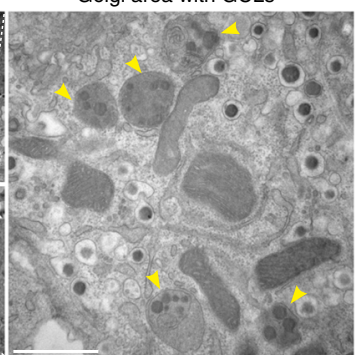

d
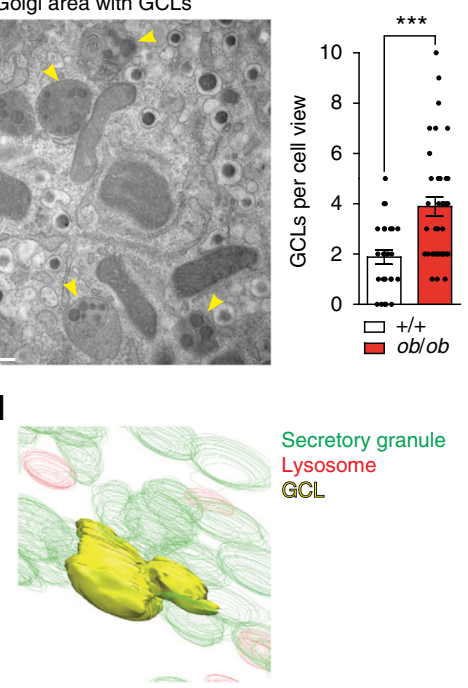

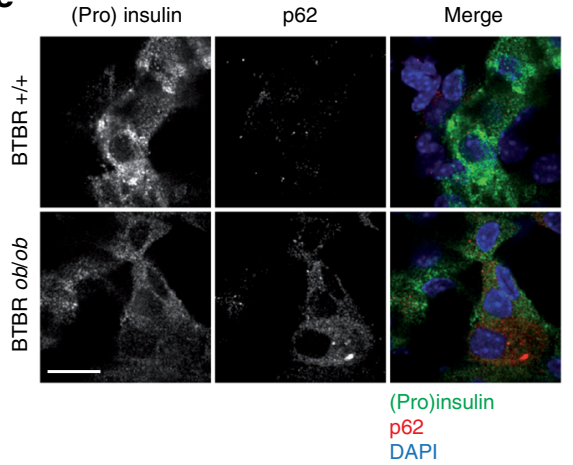

FIB-SEM of BTBR $o b / o b$

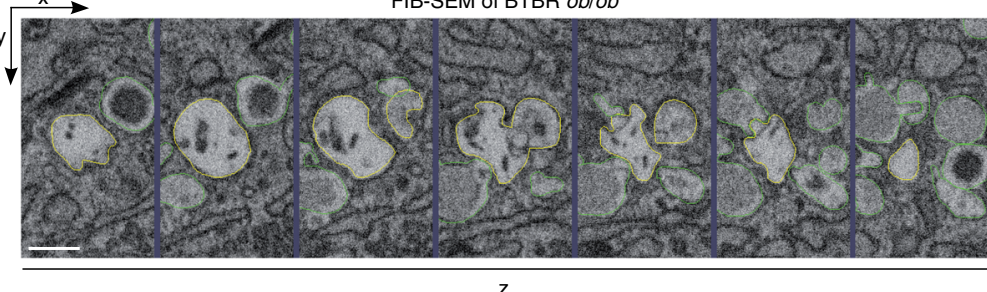

f
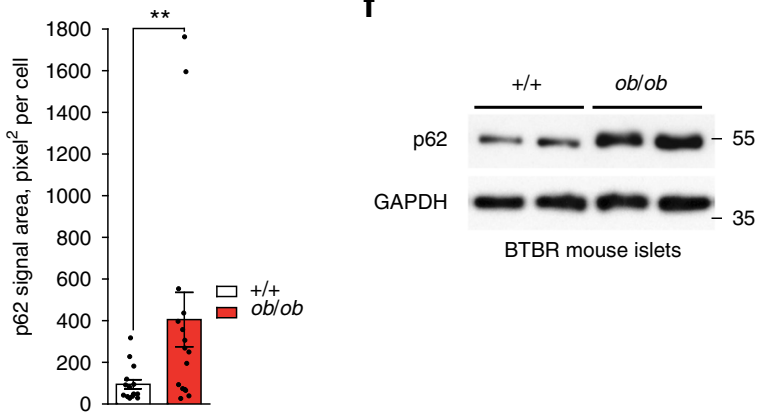

BTBR mouse islets

Fig. 3 Increased granule-containing lysosomes (GCLs) and decreased macroautophagy in diabetic mice. a Immunofluorescence (IF) of insulin + proinsulin ((pro)insulin) (green) and CD63 (red) in $\beta$ cells of 8 -week-old BTBR $+/+$ and BTBR ob/ob mice. Arrowheads point to (pro)insulin/CD63 co-localization. Scale bar, $20 \mu \mathrm{m}$. b Electron microscopy of $\beta$ cells in pancreatic islets isolated from 6 -week-old BTBR $+/+$ and BTBR ob/ob mice. Yellow arrowheads point to granule-containing lysosomes (GCLs). Scale bar, $5 \mu \mathrm{m}$; for inset: $1 \mu \mathrm{m}$. Quantification of GCLs per cell view. $N_{+/+}=25$ cells and $N_{o b / o b}=36$ cells from three mice per each group. ${ }^{\star \star} P<0.001$, Mann-Whitney U-test. c Focused lon Beam Scanning Electron Microscopy (FIB-SEM) of GCLs of 6-week-old BTBR $o b / o b$ mice. Sequential Z-slices showing GCL (yellow) and neighboring insulin granules (green). Scale bar, $0.2 \mu \mathrm{m}$. $\mathbf{d} 3 \mathrm{D}$ reconstruction of the GCL revealed by FIB-SEM in c. Detailed 3D reconstruction is shown in Supplementary Movie 3. e IF of (pro)insulin (green) and p62 (red) in $\beta$ cells of 8-week-old BTBR $+/+$ and BTBR ob/ob mice. Scale bar, $20 \mu \mathrm{m}$. Quantification of p62 signal area: area of p62 signal (pixel ${ }^{2}$ ) over a fixed signal intensity threshold. $N_{+} /+=15$; $N_{o b / o b}=16$, from three mice per each group. ${ }^{\star \star} P<0.01$, Mann-Whitney U-test. f Immunoblot of p62 using lysates of islets isolated from 8-week-old BTBR $+/+$ and BTBR ob/ob mice. GAPDH was used as a loading control. In $\mathbf{b}$ and $\mathbf{e}$ data are shown as mean \pm SEM, source data are provided as a Source Data file. (a, e) Nuclei were stained with DAPI

Increased GCLs and decreased macroautophagy in diabetic mice. We have identified enhanced SINGD in $\beta$ cell lines and primary islets treated under gluco- and lipotoxic conditions. To extend and confirm these findings in vivo we subjected mice to a metabolic stress by feeding animals a high-fat diet (HFD) for 13 weeks (Supplementary Fig. 7a, b), and analyzed the frequency of fusion events between lysosomes and SGs in $\beta$ cells. In line with above findings, EM and IF analysis of $\beta$ cells of HFD-fed mice revealed higher numbers of GCLs, as well as co-localized (pro)insulin/CD63 indicating increased SINGD (Supplementary Fig. 7c, d).

We then decided to study SINGD in a model of T2D, leptindeficient $o b / o b$ mice in a BTBR genetic background $\left(\mathrm{BTBR}^{o b / o b}\right)^{42}$. The $\mathrm{BTBR}^{\text {ob/ob }}$ mice develop severe hyperglycemia and diabetes between 6 and 8 weeks of age ${ }^{42}$ (Supplementary Fig. 8a, b). $\beta$ cells in islets of 8 -week-old BTBR $^{\text {ob/ob }}$ mice contained less (pro) insulin-positive puncta in comparison to non-diabetic control mice $\left(\mathrm{BTBR}^{+/+}\right.$mice). Strikingly, these residual puncta largely co-localized with CD63 (Fig. 3a). Furthermore, quantitative EM confirmed reduced SG abundance and revealed a marked increase in GCLs in the Golgi area of $\beta$ cells in islets of 6-week-old $\mathrm{BTBR}^{\mathrm{ob} / \mathrm{ob}}$ mice as compared to control mice (Fig. 3b). Finally, 3D EM reconstruction using Focused Ion Beam Scanning EM corroborated abundant GCLs in Golgi area of diabetic $\beta$ cells (Fig. 3c, d and Supplementary Movie 3).

As SINGD counters macroautophagy, we next analyzed macroautophagy levels in control and diabetic $\beta$ cells. p62 binds polyubiquitinated protein aggregates, mediating their clearance by autophagy ${ }^{43}$. Cytosolic accumulation of enlarged p62-positive inclusions has been described in autophagy-deficient $\beta$ cells ${ }^{44}$. Consistently, $\beta$ cells of 8 -week-old BTBR $^{o b / o b}$ mice contained large p62 puncta, suggesting compromised autophagy (Fig. 3e). Increased p62 levels in islets of $\mathrm{BTBR}^{\mathrm{ob} / \mathrm{ob}}$ mice were further corroborated by immunoblotting (Fig. 3f). Importantly, p62 protein accumulation in islets of $\mathrm{BTBR}^{\mathrm{ob} / \mathrm{ob}}$ mice was not due to increased p62 mRNA levels (Supplementary Fig. 9). 
Enhanced SINGD leads to $\beta$ cell dysfunction in diabetes. Our findings provide strong evidence for increased SINGD in diabetic $\beta$ cells. However, it is unclear whether SINGD observed upon $\mathrm{Glc} / \mathrm{Pal}$ treatment as well as in diabetic or metabolically stressed mice contributes to $\beta$ cell failure per se or represents a secondary event - for example, a removal of excessive SGs due to perturbed insulin secretion. To distinguish between these possibilities, it is necessary to follow SGs trafficking and release in conditions where SINGD is prevented. We have previously found that SINGD is controlled by PKD1: while inhibition or decreased expression of PKD1 induces SINGD, high PKD1 activity counters SINGD ${ }^{32}$. Indeed, induction of SINGD by inhibition of PKD resulted in a decrease of insulin and proinsulin in human pancreatic islets (Fig. 4a). Interestingly, consulting a published study comparing genome-wide mRNA expression in non-diabetic $\mathrm{BTBR}^{+/+}$and diabetic $\mathrm{BTBR}^{o b / o b}$ mice ${ }^{45}$, revealed a decrease in PKD1 mRNA abundance in diabetic islets (Supplementary Fig. 10). Similarly, in line with decreased PKD1 triggering SINGD in diabetic $\beta$ cells, we found a marked reduction in mRNA and protein levels of PKD1 in islets of diabetic 8-week-old $\mathrm{BTBR}^{\circ b / o b}$ mice as compared to age-matched control mice (Fig. 4b, c).

We thus decided to test whether PKD activation prevented SINGD in diabetes. Mitogen-Activated Protein Kinase (MAPK) p38 negatively regulates PKD1, and loss or reduction of p38 results in increased activity of PKD1, which counters SINGD ${ }^{32,46}$. We generated a Compound A, which selectively inhibited p $38 \delta$ and activated PKD (Fig. 4d, Supplementary Fig. 11a-c). Compound A decreased GCLs and increased SGs in Glc/Pal-treated INS1 ${ }^{\text {PGCD }}$ cells (Fig. 4e). Furthermore, phospho-PKD, proinsulin and insulin levels were increased in Compound A-treated human pancreatic islets isolated from non-diabetic donors (Fig. 4f, g, quantified in Supplementary Fig. 11d). In addition, Compound A increased insulin levels in islets isolated from T2D patients (Fig. 4h, quantified in Supplementary Fig. 11d).

Importantly, while p $38 \delta$ is abundantly expressed in pancreas, no expression of $\mathrm{p} 38 \delta$ has been detected in insulin-sensitive organs such as adipose tissue and liver ${ }^{46}$, prompting us to address the effects of Compound A on SINGD in vivo. We thus infused Compound A or a control solution into 4-week-old BTBR ${ }^{\text {oblob }}$ mice for 2 weeks using osmotic pumps and followed blood glucose levels over time. As expected, fasting blood glucose levels progressively increased in control-treated $\mathrm{BTBR}^{o b / o b}$ mice, indicating the onset of diabetes. However, glucose levels in Compound A-treated BTBR ${ }^{o b l o b}$ mice were lower than in control-treated animals indicating protection against diabetes (Fig. 4i). In strong contrast, systemic inhibition of PKD using the compound CID755673 accelerated onset of diabetes in BTBR ${ }^{\text {ob/ob }}$ mice, as evidenced by increased fasting blood glucose (Fig. 4j) and decreased insulin secretion in response to glucose injections (Supplementary Fig. 12a). Strikingly, upon islet isolation, it was evident that islets from CID755673-treated mice were pale to transparent as compared to yellow to golden islets from controltreated mice (Supplementary Fig. 12b), a phenotype hinting at the loss of insulin ${ }^{47}$. Accordingly, insulin levels were markedly decreased in islets of CID755673-treated mice (Supplementary Fig. 12c). In contrast, the numbers of GCLs were increased in $\beta$ cells of islets isolated from CID755673-treated BTBR ${ }^{o b / o b}$ mice as compared to control solution-treated BTBR ${ }^{o b / o b}$ mice (Supplementary Fig. 12d).

Collectively, these data suggest that reducing PKD activity in vivo is sufficient to trigger SINGD in $\beta$ cells, while PKD activity prevents SINGD and protects against $\beta$ cell dysfunction. Accordingly, the observed reduced PKD expression in diabetic islets is likely to contribute to enhanced SINGD in $\beta$ cells in diabetes. Even though a direct implication of enhanced SINGD to $\beta$ cell failure in vivo is to be substantiated, its link to reduced insulin content, compromised macroautophagy and insulin secretion is suggestive for such a scenario.

Stress-induced nascent granule degradation requires CD63. Our above findings reveal that reduced PKD activity triggers SINGD, while enhanced PKD activity prevents SINGD. Mechanistically, SINGD is manifested as delivery of SGs to CD63-positive lysosomes in $\mathrm{Glc} / \mathrm{Pal}$-treated $\beta$ cells and in $\beta$ cells of $\mathrm{BTBR}^{\circ b / o b}$ mice. Interestingly, we noticed that transient overexpression of CD63 resulted in a marked depletion of SGs upon Glc/Pal treatment, suggesting that CD63 might mediate SINGD (Fig. 5a). Of note, while the deletion of the lysosomal proteins LIMP2 and LAMP2 was associated with severe lysosomal dysfunction ${ }^{48,49}$, mice lacking CD63 demonstrated normal overall lysosome function ${ }^{50}$. Therefore, we decided to investigate secretion and degradation of SGs in the absence of CD63. To address the role of CD63 in SINGD, we first transfected INS1 cells with siRNA against Cd63 or non-silencing (NS) siRNA and incubated cells in the media with or without Glc/Pal for $20 \mathrm{~h}$. In control cells, transfected with NS siRNA, Glc/Pal treatment led to a decrease in (pro)insulin signals in the plasma membrane area. In contrast, SG localization remained largely unchanged in Glc/Pal-treated CD63-knockdown cells (Fig. 5b), indicating that SINGD was mediated by CD63. Furthermore, immunoblotting revealed a drop in proinsulin levels upon Glc/Pal treatment in control-transfected cells, but not in CD63-knockdown cells (Fig. 5c). To directly test whether SINGD induced by reduction of PKD requires CD63, we examined SINGD in INS1 cells stably expressing shRNA against Prkd1 (shPKD1) in the presence and absence of CD63. As expected, INS1 cells lacking PKD1 contained few SGs and displayed large CD63 puncta co-localizing with residual SGs (Fig. 5d). However, siRNA-mediated depletion of CD63 from PKD1-knockdown cells prevented loss of SGs, indicating that CD63 mediates PKD1-controlled SINGD (Fig. 5d, e). Our above findings suggest that depletion of CD63 allows SGs to escape degradation and to reach the plasma membrane, possibly for secretion. Therefore, lowering CD63 levels is expected to increase insulin secretion. To address the latter point, we next compared insulin secretion from islets derived from $C d 63$ knockout $\left(\mathrm{Cd}_{63^{-/-}} \text {mice }\right)^{50}$ and wild-type $\mathrm{Cd} 63^{+/+}$mice. Consistent with reduced SINGD, quantitative EM analysis revealed a decrease in the number of GCLs in the Golgi area, and an increase in the number of SGs associated with the plasma membrane in $\beta$ cells of islets isolated from $\mathrm{Cd}_{63^{-1-}}$ mice (Supplementary Fig. 13a, b). To test the effects of Cd63 deletion on insulin secretion upon a metabolic challenge, we also treated $\mathrm{Cd} 63^{+/+}$ and $\mathrm{Cd}_{63^{-1-}}$ islets with $\mathrm{Glc} / \mathrm{Pal}$ for $48 \mathrm{~h}$. While this treatment did not abrogate GSIS in control islets, it led to a decrease in total insulin content, most likely due to increased secretion of insulin during incubation with Glc/Pal (Supplementary Fig. 13c). Strikingly, $C d 63^{-1-}$ islets demonstrated a two-fold increase in GSIS under both control and $\mathrm{Glc} / \mathrm{Pal}$ treatment conditions (Fig. 5f). Total insulin levels were similar in metabolically challenged $C d 63^{+/+}$and $C d 63^{-/-}$islets (Supplementary Fig. 13c), suggesting that SGs escaped from SINGD in $\mathrm{Cd} 63^{-1} \beta$ cells and instead were secreted.

Taken together, our data demonstrate that $\beta$ cells developed complex machinery at the Golgi that controls targeting of insulin granules towards secretion or lysosomal degradation. Nutrient stress-imposed PKD deregulation triggers SINGD at least partially in a CD63-dependent manner. This mechanism may contribute to insulin loss, reduced secretory capacity and to decreased macroautophagy, hallmarks of $\beta$ cell dysfunction in T2D (Fig. 5g). 
a

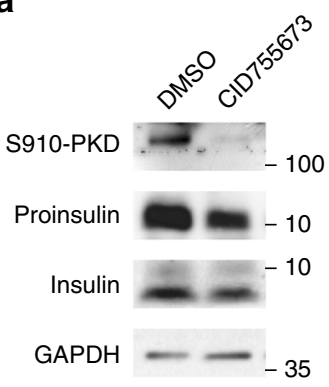

Human islets
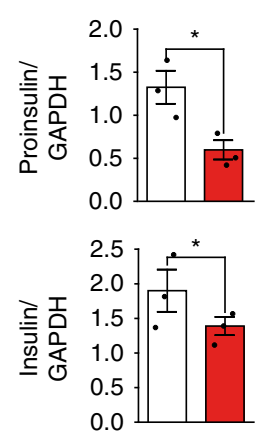

$\square$ DMSO

口 CID755673 b

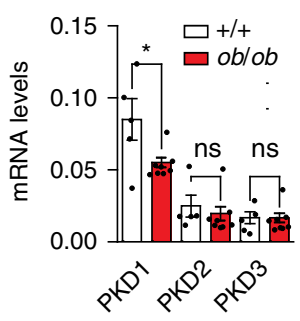

BTBR mouse islets
C

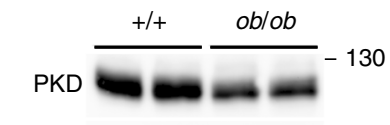

Tubulin ---55

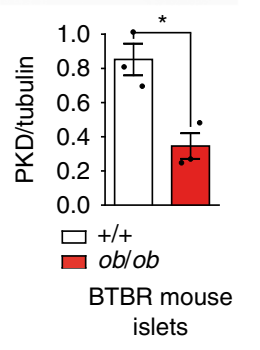

i

BTBR $o b / o b$ mice

d

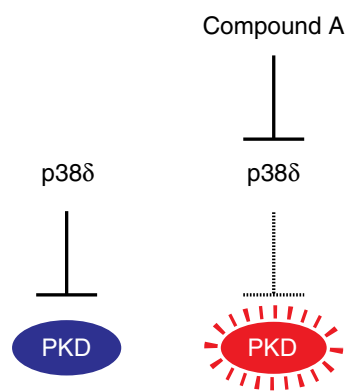

e

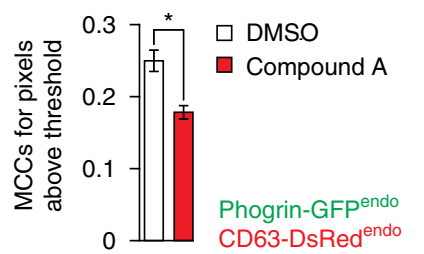

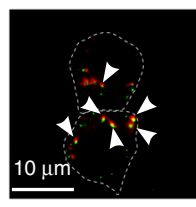

DMSO CD63-DsRed ${ }^{\text {endo }}$

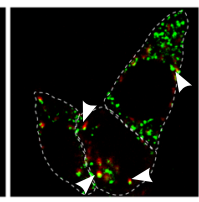

Compound $\mathrm{A}$ $\mathbf{f}$

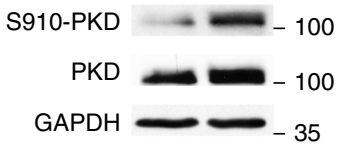

Human islets

g

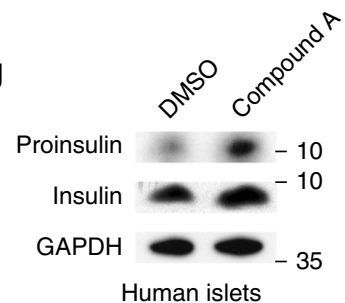

h

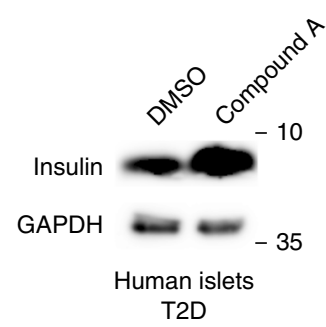

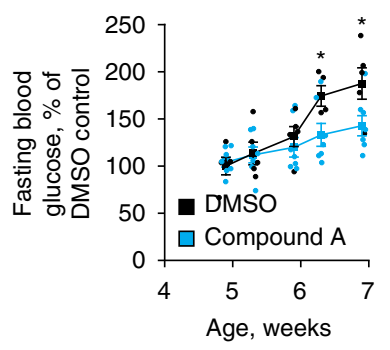

j

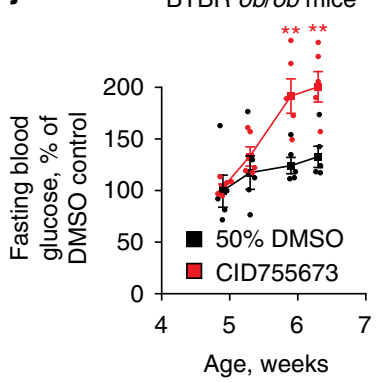

Fig. 4 SINGD contributes to $\beta$ cell dysfunction in diabetes. a Immunoblot of indicated proteins using lysates of isolated human islets treated with DMSO or PKD inhibitor CID755673 (30 $\mu \mathrm{M})$ for $24 \mathrm{~h}$. GAPDH was used as a loading control. Quantification of Proinsulin/GAPDH and Insulin/GAPDH ratios, ${ }^{\star} P<$ $0.05, N=3$, one-tailed, paired t-test. $\mathbf{b}$ Quantitative RT-PCR using mRNA isolated from pancreatic islets of 8-week-old BTBR $+/+$ and BTBR ob/ob mice using primer pairs amplifying cDNA of PKD1, PKD2 and PKD3. The expression values were normalized to housekeeping genes HPRT and GAPDH. $N_{+/+}=$ $8 ; N_{o b / o b}=5 ;{ }^{*} P<0.05$, two-tailed $t$-test. $\mathbf{c}$ Immunoblot of PKD using lysates of islets isolated from 8-week-old BTBR $+/+$ and BTBR ob/ob mice.

Tubulin was used as a loading control. Quantification of PKD/Tubulin ratio, ${ }^{\star} P<0.05, N=3$ per group, two-tailed $t$-test. $\mathbf{d}$ Compound $A$ activates $P K D$ through inhibition of p386. e Imaging of INS1 ${ }^{P G C D}$ cells endogenously expressing Phogrin-GFP(Phogrin-GFPendo) and CD63-DsRed (CD63-DsRedendo) treated with Glc/Pal for $24 \mathrm{~h}$ in the presence of DMSO or Compound A, $100 \mathrm{nM}$. Arrowheads point to Phogrin-GFPendo/CD63-DsRedendo

co-localization. Scale bar, $10 \mu \mathrm{m}$. Quantification of co-localization between CD63-DsRedendo and Phogrin-GFPendo using Manders coefficients. $N_{\text {GIc/Pal, }}$ $\mathrm{DMSO}=58 ; N_{\mathrm{Glc} / \text { Pal,Compound A }}=59$ from three independent experiments. ${ }^{\star} P<0.01$, two-tailed $t$-test. $\mathbf{f}, \mathbf{g}$ Immunoblots of indicated proteins using lysates of isolated human islets treated with DMSO or Compound A for $48 \mathrm{~h}$. GAPDH was used as a loading control. $\mathbf{h}$ Immunoblot of indicated proteins using lysates of human islets isolated from a T2D donor. Islets were treated with DMSO or Compound A for $48 \mathrm{~h}$. GAPDH was used as a loading control. i Time-course of fasting blood glucose levels of BTBR ob/ob mice of indicated ages implanted with osmotic pumps containing control solvent (DMSO) or Compound A. $N_{\text {DMSO }}=5 ; N_{\text {Compound A }}=7$; ${ }^{*} P<0.05$, two-tailed $t$-test. $\mathbf{j}$ Time-course of fasting blood glucose levels of BTBR ob/ob mice of indicated ages implanted with osmotic pumps containing control solvent (50\% DMSO) or CID755673. $N_{50 \% \text { DMSO }}=5 ; N_{\mathrm{CID755673}}=5$; ${ }^{\star \star} P<0.01$, two-tailed $t$-test. In $\mathbf{a}, \mathbf{b}, \mathbf{c}, \mathbf{e}, \mathbf{i}$, and j data are shown as mean \pm SEM, source data are provided as a Source Data file

\section{Discussion}

Our findings in $\beta$ cell lines endogenously expressing markers of SGs and lysosomes, in human and mouse islets, as well as in mouse models identified the SINGD pathway as an essential mechanism that may contribute to both loss of insulin granules and impaired macroautophagy, key features of $\beta$ cell dysfunction in $\mathrm{T}_{2} \mathrm{D}^{5,11,12,44,51-54}$. To date, several mechanisms contributing to the depletion of insulin in $\beta$ cells in T2D patients and in response to glucolipotoxicity have been reported, including impaired insulin gene transcription ${ }^{55,56}$ and mRNA stability ${ }^{57-59}$; Endoplasmic reticulum-associated degradation (ERAD) and other proteasomal degradation routes ${ }^{60}$; as well as proinsulin 
a

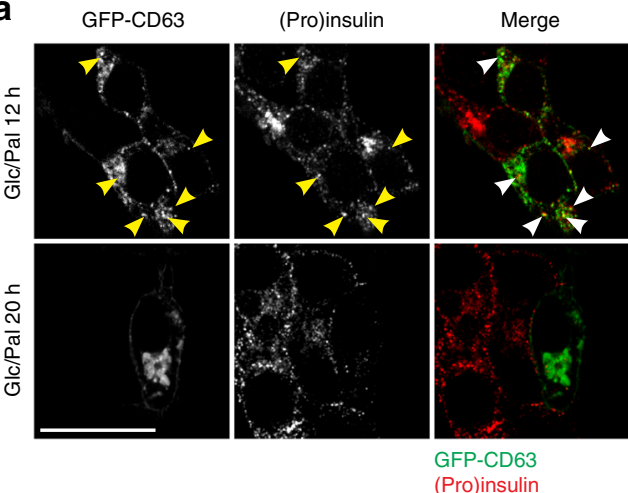

b

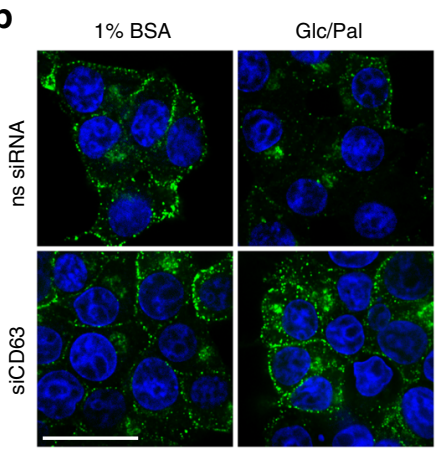

(Pro)insulin

DAPI

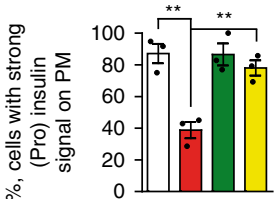

(Pro)insulin

C

d

(Pro) insulin
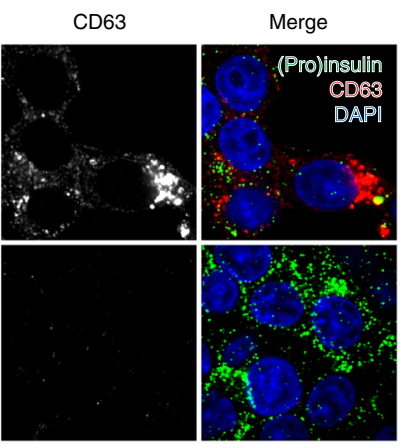

$\mathbf{e}$
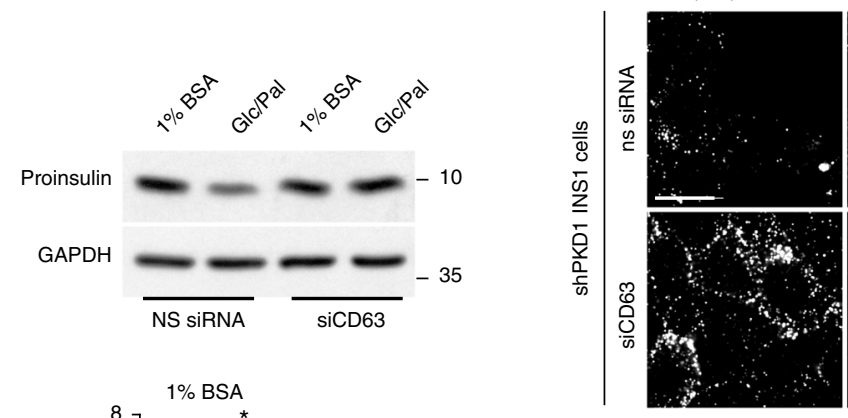
$\square 1 \%$ BSA, NS siRNA
$\square \mathrm{Glc} / \mathrm{Pal}, \mathrm{NS}$ siRNA
$\square 1 \%$ BSA, siCD63
$\square \mathrm{Glc} / \mathrm{Pal}, \mathrm{siCD63}$

$\mathbf{f}$

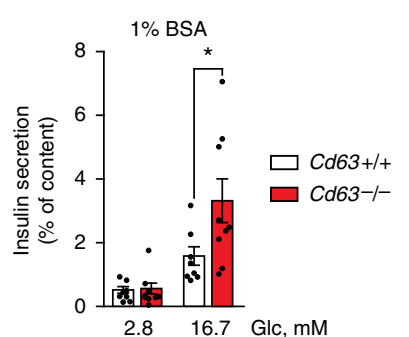

g

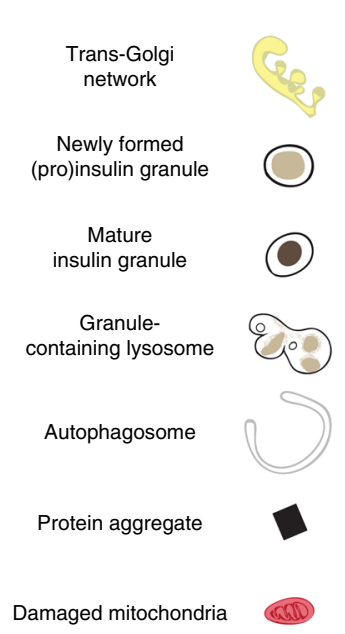

NORMAL FED STATE
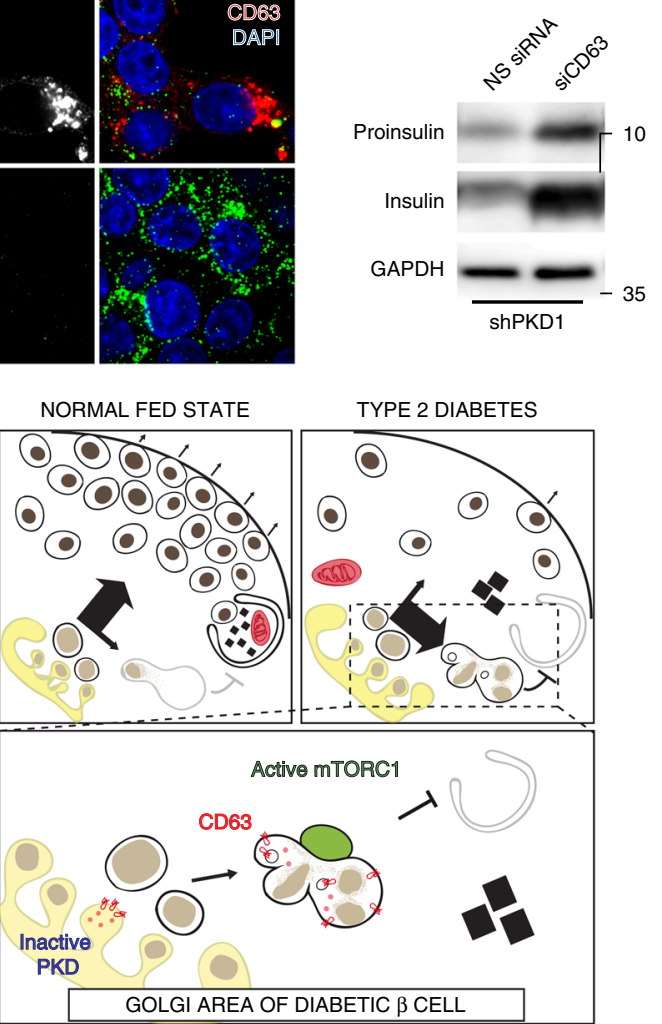

Fig. 5 Stress-induced nascent granule degradation requires CD63. a Immunofluorescence (IF) of insulin + proinsulin ("(pro)insulin") (red) in INS1 cells overexpressing GFP-CD63 (green) and treated with control (1\% BSA, $11 \mathrm{mM}$ glucose, "1\% BSA") or glucolipotoxic (1\% BSA, $33.3 \mathrm{mM}$ Glucose and $0.4 \mathrm{mM}$ Palmitate, "Glc/Pal") media for the indicated time. Arrowheads point to GFP-CD63/(pro)insulin co-localization. Scale bar, $20 \mu \mathrm{m}$. b IF of (pro)insulin (green) in INS1 cells transfected with non-silencing (ns) siRNA or siRNA against CD63 (siCD63) and treated with 1\% BSA or Glc/Pal for $20 \mathrm{~h}$. Scale bar, $20 \mu \mathrm{m}$. Quantification of (pro)insulin signals at the plasma membrane of INS1 cells treated as indicated. Three independent experiments $(N=3)$, three fields per experiment per condition, $n$ cells: $63,62,86,71$ respectively; ${ }^{\star \star} P<0.01$, two-tailed $t$-test. c Immunoblot of proinsulin using lysates of INS1 cells treated as indicated. GAPDH was used as a loading control. $\mathbf{d}$ IF of (pro)insulin (green) and CD63 (red) in INS1 cells stably expressing shRNA against Prkd1 (shPKD1), transfected with indicated siRNAs for $48 \mathrm{~h}$. Scale bar, $10 \mu \mathrm{m}$. b, d Nuclei were stained with DAPI. e Immunoblot of proinsulin and insulin using lysates from INS1 cells stably expressing shPKD1, transfected with indicated siRNAs. GAPDH was used as a loading control. f Glucose stimulated insulin secretion ( $2.8 \mathrm{mM} \mathrm{Glc}$ and $16.7 \mathrm{mM} \mathrm{Glc}$ ) of islets isolated from Cd63+/+or Cd63 -/- mice pre-treated with control (1\% BSA) or glucolipotoxic (Glc/Pal) media for $48 \mathrm{~h}$. Batches of islets were independently isolated from Cd63+/+and Cd63-/- mice; $N_{1 \% ~ B S A}=8(C d 63+/+), 9(C d 63-/-), N_{G l c} /$ Pal $=5$ $(C d 63+/+), 6(C d 63-/-),{ }^{\star} P<0.05$, two-tailed $t$-test. In $\mathbf{b}$ and $\mathbf{f}$ data are shown as mean \pm SEM, source data are provided as a Source Data file. $\mathbf{g}$ Stressinduced nascent granule degradation (SINGD) in Type 2 Diabetes (T2D). In normal fed state, PKD1 promotes secretory granule biogenesis and sorting towards secretion, SINGD is low. However, in T2D, a decrease in PKD1 leads to the CD63-dependent targeting of the newly formed granules to the CD63positive lysosomes instead of secretion. As a result, mTORC1 is recruited to the membrane of the granule-containing lysosomes, and its chronic activation leads to inhibition of the housekeeping process of macroautophagy. Thus, aberrant activation of SINGD contributes to $\beta$ cell failure in T2D 
processing and sorting ${ }^{61,62}$ and basal hypersecretion ${ }^{63}$. SINGD can now be considered as a new mechanism with potential to contribute to insulin depletion, but whether SINGD constitutes a major pathway leading to loss of insulin in T2D will require its quantification versus the other possibilities in future studies.

It is also important to mention that our results do not exclude the possibility that a small portion of byproducts of degradation might be released from cells. For example, aberrant lysosomal secretion might be an additional potential mechanism of insulin loss induced by SINGD. Interestingly, proinsulin, the major cargo in nascent granules, is released in higher amounts in diabetic patients ${ }^{64,65}$. Furthermore, recent studies identified that partially degraded granule proteins including products of insulin degradation might be released from diabetic $\beta$ cells ${ }^{66,67}$.

Several potential mechanisms leading to impaired $\beta$ cell macroautophagy in T2D have been proposed, including defects in autophagosome/lysosome fusion ${ }^{13,16,18,19}$ and more broadly, primary defects in lysosomal function ${ }^{20}$. Our data hint at impaired $\beta$ cell macroautophagy in T2D as a consequence of mTOR-dependent reduction in autophagosome formation in response to increased SINGD. Importantly, recent studies have also established the role of mTOR in more distal steps of macroautophagy 68,69 suggesting that SINGD-mTOR-induced inhibition of macroautophagy may not be limited to reducing autophagosome formation.

Genetically induced mTORC1 hyperactivity has been reported to be deleterious and to promote onset of diabetes in vivo due to compromised macroautophagy ${ }^{52}$. Importantly, chronic mTORC1 inactivation also led to rapid onset or aggravation of diabetes ${ }^{70,71}$. Mechanistically, mTORC1 inactivation mainly impaired $\beta$ cell compensation in response to metabolic stress. Together with our findings, these data argue for a model in which nutrientdependent switching between high and low mTORC1 activity promoting $\beta$ cell growth/proliferation or macroautophagy, respectively, is crucial to maintain $\beta$ cell function ${ }^{72}$. This model is also supported by a recent report demonstrating that intermittent fasting promoted resistance against ageing and diabetes ${ }^{73}$.

Our previous work unveiled that $\beta$ cells utilize SINGD in response to nutrient deprivation, thereby suppressing macroautophagy ${ }^{32}$. An aberrant activation of SINGD in diabetic conditions somehow parallels one of the hallmarks of T2D, which is constitutive induction of fasting-related processes, i.e., gluconeogenesis and lipolysis, in a situation of nutrient overload. Although the latter phenomena are related to insulin resistance ${ }^{74}$ as well as hyperglucagonaemia ${ }^{75}$, it remains unclear, how nutrient deprivation and nutrient excess both can trigger SINGD in $\beta$ cells. It will be important to understand mechanistically how these seemingly opposing nutrient stresses both converge into reduced PKD activity at the TGN triggering SINGD.

Studies from our group and others have previously implicated perturbed Protein Kinase D activity in the pathogenesis of T2D ${ }^{46,76-79}$. Furthermore, PRKD1 (human PKD) was identified in several genome-wide associated studies as a Body Mass Indexassociated locus ${ }^{80-82}$ as well as T2D-associated locus ${ }^{83}$. Our findings establish decreased PKD as a trigger for SINGD in diabetic $\beta$ cells of mice and humans, thus suggesting a mechanistic explanation for compromised PKD activity contributing to T2D.

Our study also provides evidence for tetraspanin CD63 to be an essential component for targeting insulin granules to the lysosomes (Fig. 5g). Although high CD63 levels induce SINGD, depletion of CD63 in $\beta$ cell lines and in islets prevents SINGD, restoring the pool of SGs. CD63 has been previously implicated in the sorting and targeting of various substrates to the endolysosomal system ${ }^{84-86}$. How does PKD activity limit CD63-mediated SINGD? According to one potential scenario, granules routed to lysosomes may lack PKD-dependent signals generated at the
Golgi that prevent CD63-dependent fusion with lysosomes. In other words, CD63 might be a necessary component on lysosomes independent of PKD activity but whether or not fusion occurs might be dictated by PKD-dependent signals on the insulin granule. Alternatively, PKD may also more directly inhibit CD63 function on lysosomes or other lysosomal proteins that are required for fusion to occur. Future efforts will be necessary to test the above models. Hence, unraveling of detailed molecular mechanisms of SINGD will be required to address a more direct physiological impact of the SINGD pathway on $\beta$ cell function and glucose homeostasis in vivo.

What is the place of SINGD in the context of known and putative degradation mechanisms? Our findings demonstrate that SINGD occurs via direct fusion of nascent SGs with lysosomes. Seminal electron microscopy studies performed in various secretory cells, including cells of the anterior pituitary gland ${ }^{87}$, pancreatic $\alpha$ cells ${ }^{88}$, and $\beta$ cells ${ }^{89}$ had presented strong evidence for selective fusion between secretory granules and lysosomes, which in some cases correlated with conditions of perturbed secretion. This process was designated as crinophagy to distinguish it from canonical autophagy (macroautophagy) ${ }^{90}$. Csizmadia et al. have recently presented an extensive analysis of molecular mechanisms of crinophagy in the salivary gland of Drosophila, further highlighting the evolutionary importance of this pathway independent of macroautophagy ${ }^{91}$. The relevance of these findings in the context of p38 $/ \mathrm{PKD}$ - and CD63dependent SINGD remains to be established.

Recently, a study by Riahi et al. performed in $\beta$ cells overexpressing markers of SGs, macroautophagy as well as lysosomes claimed that macroautophagy was a major pathway through which insulin granules were degraded ${ }^{30}$. According to our data, this is not the case for SINGD. Yet, none of our findings contradicts the possibility that insulin SGs may eventually be delivered to lysosomes via autophagosomes along with other cytosolic material. Indeed, double-membrane autophagosomes containing SGs have been reported to occur in $\beta$ cells $^{28}$. Importantly however, a large body of evidence in our and other studies demonstrates that targeting of secretory granules to lysosomes can occur independent of canonical macroautophagy $25,26,28,31,32,87,91$. Furthermore, if macroautophagy was mainly responsible for SGs degradation, macroautophagy-deficient $\beta$ cells would be expected to accumulate SGs, but not to lose them as observed by Riahi et al. and in other studies $5,30,44$. In addition, consistent with our own findings, Riahi et al. observed that treatment of $\beta$ cells with BafA1 resulted in marked increase in co-localization of insulin granules with lysosomes. Among other effects, BafA1 blocks fusion of autophagosomes with lysosomes ${ }^{37,38}$ (Supplementary Fig. 4h) which prevents autophagosomal delivery of insulin granules to lysosomes. Finally, our data strongly indicate that SINGD induced by glucolipotoxicity does not require the macroautophagy machinery, as silencing of major macroautophagy genes atg 5 and beclin 1 has virtually no effect on SINGD.

Interestingly, a recent study has reported that secretory granules might be delivered to lysosomes via autophagosome-like structures originating at the Golgi in $\beta$ cells devoid of factors required for autophagosome formation (ATG5 and ATG7) ${ }^{31}$. Strikingly, both Golgi membrane-associated degradation (GOMED) and SINGD occur in lysosomes adjacent to the Golgi area independent of the classical macroautophagy machinery. However, three important aspects distinguish GOMED from direct fusion of insulin granules with lysosomes as a mechanism underlying SINGD: (1) GOMED is a feature in cells lacking the canonical macroautophagy machinery. (2) Direct fusion of insulin granules with lysosomes does not rely on autophagosomelike intermediates to target SGs to lysosomes. (3) GOMED is not restricted to degradation of insulin granules but is also a way to 
target for example mitochondria to lysosomal degradation in the absence of canonical macroautophagy.

Collectively, the delivery of insulin granules to lysosomes can be achieved by several mechanisms including macroautophagy and macroautophagy-like pathways. However, our findings indicate that starvation- or metabolic stress-induced SINGD is macroautophagy-independent and even inhibits macroautophagy through mTORC1 activation.

Our current study together with our and others' published evidence $^{31,32}$ demonstrate that $\beta$ cells tightly control the fate of newly formed cargo-containing carriers. On the cellular level, this means that SGs on their route to the plasma membrane need to bypass the lysosomes in the Golgi area. In light of the well-known mechanisms of protein quality control in endoplasmic reticulum (ERAD), it is tempting to speculate that the SINGD pathway might play a similar role at the Golgi: SGs incapable of interacting with the secretory trafficking machinery, or containing wrong cargos, etc. might need to be efficiently cleared to prevent 'clogging' of the Golgi area with non-functional byproducts of secretion. Our data indicate that SINGD is relatively low under basal conditions, and it is markedly enhanced upon nutrient stress, leading to depletion of SGs. The comparison between SGs targeted for degradation and SGs involved in secretion remains to be done. Nonetheless, in line with this hypothesis, enhanced degradation of SGs was previously observed in models of perturbed insulin release, and was therefore considered as a means to counter-balance SG accumulation ${ }^{28,92}$. Importantly, however, our study suggests that aberrant SINGD might be directly involved in the pathogenesis of T2D: first, we observed an exacerbation of T2D upon triggering SINGD and second, preventing SINGD by keeping PKD active or by reducing the levels of CD63 restored the SG pool and insulin secretion.

Altogether, our findings demonstrate that, under diabetic conditions, stress-induced nascent granule degradation (SINGD) interferes with the protective role of macroautophagy in $\beta$ cells, which may contribute to premature $\beta$ cell failure in T2D. Our study also links lysosomal degradation of SGs to $\beta$ cell failure and provides the first steps towards understanding the molecular mechanisms of the SINGD pathway, suggesting potential therapeutic avenues in T2D.

\section{Methods \\ Common reagents. Reagents were obtained from the following sources: HRP- conjugated anti-mouse and anti-rabbit secondary antibodies from IGBMC; oligo- nucleotides, type V Collagenase solution, Bafilomycin Al from Sigma Aldrich; Mowiol from Calbiochem; cOmplete Protease inhibitor cocktail tablets from Roche; Rapamycin and CID755673 from Tocris Biosciences; 4',6-diamidino-2- phenylindole (DAPI), Image-iT ${ }^{\circledR}$ FX signal enhancer and Alexa $-488,-568$, and -647 conjugated secondary antibodies from Life Technologies; Osmotic pumps from Alzet ${ }^{\oplus}$. Golgi-CFP and GFP-CD63 were obtained from Addgene. Golgi-CFP was a gift from Alexandra Newton, University of California San Diego (Addgene plasmid \# 1487393). It contains the sequence encoding the amino-terminal 33 residues of Golgi-associated endothelial nitric-oxide synthase in frame with the $5^{\prime}$ - end of CFP. GFP-CD63 (CD63-pEGFP C2) was a gift from Paul Luzio (Addgene plasmid \# 62964). It contains full-length human CD63 cloned into pEGFP C2.}

Primary antibodies used. Fig. 1a: insulin, Cell Signaling Technology (CST), produced in rabbit (4590) (1/100). Giantin G1/133, Enzo life science monoclonal, mouse, ALX-804-600-C100) (1/1000).

Fig. 1b: insulin, CST, produced in rabbit (4590) (1/100). Anti rat-CD63 produced in mouse Biorad (AD-1), formerly Serotec (1/100).

Fig. 2a and Supplementary Movie 2: anti rat-CD63 produced in mouse Biorad (AD-1), formerly Serotec (1/100). mTOR, 7C10, CST, produced in rabbit (2983) $(1 / 200)$.

Fig. 2b: phospho-ULK1 (Ser757), CST produced in rabbit (6888).

Fig. 2d: LC3B antibodies: (1) 2G6, produced in mouse, Nanotools (1/1000) and (2) Novus, produced in rabbit, (NB100-2220) (1/1000). GAPDH, Sigma, produced in rabbit (G9545) $(1 / 10,000)$.

Fig. 3a: insulin, CST, produced in mouse L6B10 (8138) (1/100). CD63, produced in rabbit, from $P$. Saftig $(1 / 100)$
Fig. 3e: insulin, CST, produced in rabbit 4590 (1/100). p62, Progen, produced in guinea pig (GP62-C) (1/100).

Fig. 3f: p62, progen, produced in guinea pig (GP62-C) (1/1000). GAPDH Sigma produced in rabbit (G9545) (1/10,000).

Fig. 4a: anti-phospho-PRKD1 (pSer910), Sigma, produced in rabbit

(SAB4300075) (1/1000). Tubulin, Sigma, produced in mouse (T9026) $(1 / 10,000)$. Insulin, Sigma, produced in guinea Pig (I8510).

Fig. 4c: PKD/PKC $\mu$ Antibody, CST, rabbit (2052) (1/1000). Tubulin, Sigma, produced in mouse (T9026) $(1 / 10,000)$.

Fig. 4f: anti-phospho-PRKD1 (pSer910), Sigma, produced in rabbit (SAB4300075) (1/1000). PKD/PKC $\mu$ Antibody, CST, rabbit (2052) (1/1000). GAPDH Sigma produced in Rabbit (G9545) (1/10,000).

Fig. 4g, h: insulin, Sigma, produced in Guinea Pig (I8510). GAPDH Sigma produced in Rabbit (G9545) (1/10,000).

Fig. 5a: insulin, CST, produced in Rabbit (4590) (1/100).

Fig. 5b: insulin, Sigma, produced in mouse K36AC10 (I2018) (1/1000).

Fig. 5c: C-peptide I, produced in mouse, Biorad, Formerly Serotec (MCA2857)

(1/1000). GAPDH Sigma produced in Rabbit (G9545) (1/10,000).

Fig. 5d: insulin, CST, produced in Rabbit (4590) (1/100). Anti rat-CD63 produced in Mouse Biorad (AD-1), formerly Serotec (1/100).

Fig. 5e: insulin, Sigma, produced in Guinea Pig (I8510). GAPDH Sigma produced in Rabbit (G9545) (1/10,000).

Supplementary Fig. 1a: insulin, Sigma, produced in mouse K36AC10 (I2018) (1/ 1000). Insulin, CST, produced in Rabbit (4590) (1/100)

Supplementary Fig. 1b: insulin, CST, produced in Mouse L6B10 (8138) (1/100). Lamp2, Invitrogen, produced in Rabbit (1/700).

Supplementary Fig. 2c: GFP, ThermoFisher Scientific, produced in rabbit, A6455. GAPDH, Sigma, produced in Rabbit (G9545) (1/10,000).

Supplementary Fig. 2e: insulin, CST, produced in Rabbit (4590) (1/100).

Supplementary Fig. 2i: anti rat-CD63 produced in Mouse Biorad (AD-1),

formerly Serotec $(1 / 100)$

Supplementary Fig. 4a: insulin, CST, produced in Rabbit (4590) (1/100).

Supplementary Fig. 4b: an antibody against Phogrin was a generous gift from

John Hutton and Howard Davidson (University of Colorado) (1/1000).

Supplementary Fig. 4c: Beclin-1, CST, produced in rabbit (3495) (1/1000).

ATG5, CST, produced in rabbit (12994) (1/1000). GAPDH Sigma produced in

Rabbit (G9545) (1/10,000).

Supplementary Fig. 4i: LC3B antibody (PM036) from MBL.

Supplementary Fig. 7d: insulin, CST, produced in Mouse L6B10 (8138) (1/100). CD63, produced in Rabbit, from P. Saftig (1/100).

Mice. BTBR $o b /+{ }^{42}$ (BTBR background) mice were obtained from Charles River and were maintained by heterozygous breeding to generate $+/+$ and $o b / o b$ littermates. $C d 63^{-1-}$ mice (C57BL/6 $\times 129 / \mathrm{SV}$ background) were generated and described previously ${ }^{50}$. Mice were housed under controlled temperature on a $12-\mathrm{h}$ light/dark cycle with unrestricted access to water and standard laboratory chow. Maintenance and animal experimentation were in accordance with the local ethical committee (Com'Eth) in compliance with the European legislation on care and use of laboratory animals.

Mice experiments. Male mice were used in all experiments. For the High Fat Diet (HFD) experiments, mice (C57BL/6 × 129/SV background) were put under HFD (d12492i from Research $\operatorname{diet}^{\circledR}$ ) for 13 weeks starting at 5 weeks of age and were monitored weekly. For pharmacological treatments, mice were implanted with osmotic pumps according to the manufacturer protocol at 4 weeks of age under anesthesia induced by $3-4 \%$ Isoflurane inhalation and maintained with $1.5 \%$ Isoflurane. CID755676 was dissolved at $10 \mathrm{mg} / \mathrm{ml}$ in $50 \%$ DMSO solution. Compound A was diluted in DMSO. For Glucose Stimulated Insulin Secretion (GSIS) test, the mice were fasted and intraperitoneally injected with a glucose solution $(2 \mathrm{~g} / \mathrm{kg}$ body weight). $100 \mu$ l blood samples were repeatedly taken from the tail tip. Samples were immediately centrifuged and plasma was stored at $-20^{\circ} \mathrm{C}$. Insulin was measured in $25 \mu \mathrm{l}$ plasma using Insulin Ultrasensitive ELISA (ALPCO).

Molecular cloning and INS ${ }^{P G C D}$ knock-in cell line generation. The modified repair plasmid was generated by amplifying the rat CD63 C-terminal sequence with modified primers complementary for the DsRed sequence. The DsRed sequence was amplified from a DsRed containing plasmid with modified primers complementary for the CD63 C-terminal region. 3 PCR fragments were put together with a SmaI digested opened puc57 plasmid and treated with ExoIII nuclease which selectively removes the $3^{\prime}$ end of double stranded DNA fragment and allow complementary annealing between the PCR fragments and the puc57 plasmid. The recombinant plasmid was transformed and amplified in STBL3 competent E.coli and verified by Sanger sequencing (GATC). The same strategy was used to generate the Phogrin-eGFP mutants (PTPRN2-eGFP).

Primers sequence $\left(5^{\prime}-3^{\prime}\right)$ of puc57-CD63 HR1 Fw:

CGAATGCATCTAGATATGGGATCCTGACTGGGCCAGAGCAAGCCCTT

TAAAT; CD63 HR1-DsRed Rv:

GTCCTCGGTGTTGTCCATTACTTCGTAGCCACTCCGGATAC; CD63 HR1-DsRed Fw: 
GAGTGGCTACGAAGTAATGGACAACACCGAGGACGTCATCAAGG; DsRed-CD63 HR2 Rv:

TCAGACACGCCCCCACCTCACTGGGAGCCGGAGTGGC;

DsRed-CD63 HR2 Fw:

CCACTCCGGCTCCAGTGAGGTGGGGGCGTGTCTGAGCTC

CD63 HR2-puc57 Rv:

GCCTCTGCAGTCGACGGGCCCGGGCTTTACAGCAA-

GAGGCTGGTTTCGG; puc57-PTPRN2 HR1 Fw:

CGAATGCATCTAGATATCGGATCCAGGCAGGGGCTGGTTCTGATTG

CAC; PTPRN2 HR1-eGFP Rv:

TGAACAGCTCCTCGCCCTTGCTCACCTGGGGAAGGGCCTTCAGGAT

GGCA; PTPRN2 HR1-eGFP Fw:

TGCCATCCTGAAGGCCCTTCCCCAGGTGAGCAAGGGCGAGGAGC

TGTTCA; eGFP-PTPRN2 HR2 Rv:

TCCCGTCAGCTCCAGCTTCGGTGCCTACTTGTACAGCTCGTCCATGC

CGA; eGFP-PTPRN2 HR2 Fw:

TCGGCATGGACGAGCTGTACAAGTAGGCACCGAAGCTGGAGCTGACG

GGA PTPRN2 HR2-puc57 Rv:

GCCTCTGCAGTCGACGGGCCCGGGCAAACTGGAGCATCCAGTGAGA

ATGT. The specific gRNAs were designed (crispr.mit.edu) and purchased at Sigma Aldrich. They were cloned inside the Cas9-containing pX330 plasmid according to the manufacturer protocols (Addgene). Additional nucleotides for correct insertion after BbsI digestion were inserted.

gRNA sequences $\left(5^{\prime}-3^{\prime}\right)$ of CD63 KI gRNA 1 Fw:

caccGGGCTACGAAGTAATGTAGGGTGG:

CD63 KI gRNA 1 Rv:

aaacCCACCCTACATTACTTCGTAGCCC;

Phogrin KI gRNA 5 Fw:

caccGTCCCCAGTAGGCACCGAAGC;

Phogrin KI gRNA 5 Rv:

aaacGCTTCGGTGCCTACTGGGGAC.

To generate knock-in cell line, INS1 cells were transfected with corresponding plasmids $(20 \mu \mathrm{g}$ each). To select single clones, cells were diluted at 70 cells $/ 10 \mathrm{ml}$ concentration and spread over 96-well plates, with $100 \mu \mathrm{l}$ of media per well. Clones were screened by PCR.

Primers sequence $\left(5^{\prime}-3^{\prime}\right)$ of Sequencing CD63-Dsred Fw:

CGAATGCATCTAGATATCGGATCCTGTGGTCATCATTGCAGTGGGT; Sequencing CD63-DsRed Rv:

TCTGCAGTCGACGGGCCCGGGGTACAAGGACAACATGCTCAACGAC; Sequencing Phogrin-GFP Fw:

\section{CGAATGCATCTAGATATCGGATCCTCAACATAAAGAGCAGAGGCCAAC;} Sequencing Phogrin-GFP Rv:

GCCTCTGCAGTCGACGGGCCCGGGCAGGTGGTAAGCCCAACGCCCAAA

Clones positive for the insertion were then checked by Sanger sequencing

(GATC), immunofluorescence analysis and/or immunoblot.

Cell lines and transfections. INS1 cells were maintained in RPMI-1640 supplemented with $10 \%$ FCS, $10 \mathrm{mM}$ Hepes, $1 \mathrm{mM} \mathrm{PyrNa}, 2 \mathrm{mM}$ glutamine, $50 \mu \mathrm{M} \beta$ mercaptoethanol, penicillin, and streptomycin. Cells were transfected by using Amaxa Nucleofector (Lonza) according to the manufacturer's protocol using the T027 program.

Islet isolation and culture. Mouse islets were isolated by type $\mathrm{V}$ collagenase (Sigma Aldrich) digestion and Histopaque density gradient centrifugation and allowed to recover overnight in RPMI-1640 medium 11, $1 \mathrm{mM}$ glucose, supplemented with $10 \%$ (vol/vol) FBS, $10 \mathrm{mM}$ Hepes, $1 \mathrm{mM}$ PyrNa, $2 \mathrm{mM}$ glutamine, $50 \mu \mathrm{M} \beta$-mercaptoethanol, and Penicillin/streptomycin mix. Isolated islets from non-diabetic human cadaveric donors (authorization number for the human tissue protocol: PFS12-013) were provided by the Integrated Islets European consortium for islet transplantation and the Centre Européen d'étude du diabète (CEED). Human islets were cultured in CMRL medium.

Insulin static incubations. For static incubations, batches of 10 islets each were first kept in KRB solution containing $0.1 \%$ (wt/vol.) BSA and $2.8 \mathrm{mM}$ glucose for $40 \mathrm{~min}$ ( 2 incubations $20 \mathrm{~min}$ each) at $37^{\circ} \mathrm{C}$, then incubated for $1 \mathrm{~h}$ in the presence of 2.8 or $16.7 \mathrm{mM}$ of glucose. Each condition was run in triplicate. Intracellular insulin content was measured after acid-alcohol extraction. Insulin levels were measured by ELISA (ALPCO, Eurobio, Courtaboeuf, France).

Lysates preparation and immunoblotting. Tissues or cells were rinsed once with ice-cold PBS and lysed in ice-cold lysis buffer (50 mM Tris (pH 7.5), $50 \mathrm{mM} \mathrm{NaCl}$, $0.5 \%$ Triton X-100, 0.5\% NP40 substitute, 5 mM EGTA, 5 mM EDTA, $20 \mathrm{mM} \mathrm{NaF}$, $25 \mathrm{mM} \beta$-glycerophosphate, $1 \mathrm{mM}$ PMSF, $0.1 \mathrm{mM} \mathrm{NaVO3}$, 1x cOmplete protease inhibitor (Roche)). The soluble fractions of cell lysates were isolated by centrifugation at $14,000 \times g$ for $10 \mathrm{~min}$. Protein samples were separated by using SDSPAGE or TRICINE-SDS-PAGE and transferred to PVDF membranes. Membranes were blocked at room temperature for $1 \mathrm{~h}$ with $5 \%$ skimmed milk in TBST $(0.05 \%$
Tween20). Primary antibody incubation was done overnight at $4{ }^{\circ} \mathrm{C}$. The membranes were washed three times with TBST and incubated with the secondary antibody for $1 \mathrm{~h}$ and finally washed again three times with TBST. Proteins were visualized with Luminata Forte ECL (Millipore) on the AI600 camera (GE health) or using X-ray films. Uncropped immunoblots are shown in Supplementary Fig. 14

qPCR analysis. Tissues or cells were homogenized in TRIzol reagent (Sigma). After phase separation by centrifugation, the aqueous phase was transferred into a new tube and RNA was precipitated by $75 \%$ ethanol. cDNA was synthesized with Oligo dT primers using SuperScript III forst- strand cDNA synthesis kit (Invitrogen) according to manufacturer's protocol. Quantitative real-time PCR was performed using SYBR Green (Roche) on a LightCycler 480 (Roche). The expression values were individually normalized to housekeeping genes HPRT and GAPDH.

Primers sequence $\left(5^{\prime}-3^{\prime}\right)$ of HPRT mouse fw:

AGTCCCAGCGTCGTGATTAG;

HPRT mouse rv:

TTTCCAAATCCTCGGCATAATGA;

GAPDH mouse fw: TTGGCCGTATTGGGCGCCTG:

GAPDH mouse rv: CACCCTTCAAGTGGGCCCCG;

PrKD1 mouse fw: CGTGGTTAACCCATCAAGTT;

PrKD1 mouse rv: AATTCCAAACTGTCCGGAAC

PrKD2 mouse fw: GCAACTAGCCTGTTCTATCGTG;

PrKD2 mouse rv: AGAGCAGGATCTTGTCGTACA;

PrKD3 mouse fw: CAGTGGAAGACTTCCAGATCC;

PrKD3 mouse rv: GAATCTTGAAGGCACATCGTTTATG.

P62 mouse fw: GCTGCCCTATACCCACATCT

P62 mouse rv: CGCCTTCATCCGAGAAAC

Immunofluorescence microscopy. INS1 cells were plated on 9-15 mm glass coverslips (Menzel-Glaser) in 24-well tissue culture plates and allowed to grow for $24 \mathrm{~h}$. In the end of experiment the cells were washed once with PBS and fixed for 20 min with $4 \%$ paraformaldehyde (PFA) in PBS at room temperature. The coverslips were rinsed three times with PBS and permeabilized with $0.1 \%$ Triton X-100 in PBS for 5 min. After rinsing three times with PBS, the coverslips were incubated in Image-iT ${ }^{\circledast}$ Fx Signal enhancer for $30 \mathrm{~min}$, rinsed once with PBS and incubated for $30 \mathrm{~min}$ in blocking buffer (3\% BSA in PBS). Coverslips were subsequently incubated with primary antibodies in blocking buffer for $1 \mathrm{~h}$ at room temperature, rinsed three times in PBS and incubated with the secondary antibodies, diluted in blocking buffer, for $40 \mathrm{~min}$ at room temperature in the dark. After incubation, coverslips were rinsed three times with PBS (with DAPI added in the second washing step, when present) and mounted on glass slides using Vectashield, ProLong Gold or Mowiol and imaged with a $\times 100$ or $\times 63$ objective using fluorescence microscope of confocal Leica spinning Disk Andor/Yokogawa or Nikon Ti PFS.

Pancreata were fixed by intracardiac perfusion with PFA $4 \%$ in PBS. Tissues were post-fixed overnight at $4{ }^{\circ} \mathrm{C}$ in the same solution, cryoprotected in $30 \%$ sucrose in PBS solution, embedded in Cryomatrix (Thermo scientific) and kept at $-80^{\circ} \mathrm{C}$. Pancreas sections were cut with a cryostat (CM3050, Leica) and mounted on Superfrost Ultra Plus slides (Menzel-Glaser). Cryosections $(10 \mu \mathrm{m})$ were incubated for $2 \mathrm{~h}$ at room temperature in the blocking solution (PBS/5\% Normal Goat serum $/ 0.3 \%$ Triton X-100), then left overnight at $4{ }^{\circ} \mathrm{C}$, in a wet chamber, with primary antibodies. After 3 washes in PBS, the sections were blocked for $15 \mathrm{~min}$ at room temperature in the blocking solution and incubated for $2 \mathrm{~h}$ at room temperature, in wet chamber, with secondary antibodies diluted in blocking solution. After three washes in PBS, one wash in milliQ water, the slides were dried and mounted in Mowiol (Calbiochem) containing DAPI $(0.5 \mu \mathrm{M})$. Images were taken using confocal microscope Leica Spinning Disk Andor/Yokogawa or Nikon Ti PFS.

Live microscopy. INS ${ }^{\mathrm{PGCD}}$ were cultured overnight on $60 \mu$-Dish $35 \mathrm{~mm}$, high Glass bottom from Ibidi ${ }^{\oplus}$, processed as described in the text and imaged with the Nikon Ti PFS Spinning Disk.

Co-localization analysis. To assess pixel co-localization, we used: (a) The automatic threshold Pearson's analysis with the Fiji plugin Co-localization thresholds. The plugin generated the co-localized pixel maps, a scatter plot as well as the Pearson's correlation coefficient; (b) The JACOP plugin (Fiji) to determine Manders coefficients.

Transmission electron microscopy. For transmission electron microscopy (TEM), cells and primary islets were fixed $1 \mathrm{~h}$ with $2.5 \%$ glutaraldehyde and $2.5 \%$ formaldehyde in $0.1 \mathrm{M}$ cacodylate buffer, rinsed in buffer and followed by $1 \mathrm{~h}$ postfixation in $1 \%$ osmium tetroxide [OsO4] reduced by $1 \%$ potassium ferricyanure $[\mathrm{K} 3 \mathrm{Fe}(\mathrm{CN}) 6]$ in dark on ice. After extensive rinses in distilled water, samples were then stained first by in $1 \%$ tannic acid, followed after rinses by $1 \%$ uranyl acetate, for $1 \mathrm{~h}$ on ice each, and rinsed in water. Samples were dehydrated with increasing concentrations of ethanol $(50 \%, 70 \%, 90 \%$, and $3 \times 100 \%)$, and embedded with a graded series of epoxy resin. The blocs were finally polymerized at $60{ }^{\circ} \mathrm{C}$ for $48 \mathrm{~h}$. Ultrathin sections $(50 \mathrm{~nm})$ were picked up on $1 \%$ pioloform 
coated copper slot grids and observed with a Philips CM12 operated at $80 \mathrm{kV}$ equipped with an Orius SC100 CDD camera (Gatan, Pleasanton, USA).

Quantitative analysis of granule-containing lysosomes, lysosomes, and insulin granules of INS1 cells and primary islets has been assessed by TEM as in Goginashvili et al. ${ }^{32}$. In brief, the areas of cytoplasm and Golgi were evaluated by stereological approach using $\times 5600$ magnification with ImageJ-based open source Fiji software package. The numbers of compartments were quantified using $\times 15,000$ or $\times 19,500$ magnification. Data from three independent experiments were expressed as mean \pm SEM. All quantitative analyses relied on systematic uniform random sampling.

CLEM. Correlative Light and Electron Microscopy (CLEM) was performed as in Lenormand et al. ${ }^{94}$. In brief, INS1 cells were cultured on laser micro-patterned Aclar supports. In the end of experiment, the cells were fixed in PFA 4\%, Glutaraldehyde $0.5 \%$ in $0.1 \mathrm{M}$ sodium cacodylate buffer $0.1 \mathrm{M}$ for $20 \mathrm{~min}$ at room temperature, followed by incubation in sodium cacodylate $0.1 \mathrm{M}$. Cells of interest were selected, precisely located and imaged by fluorescence confocal microscopy using Leica Spinning Disk Andor/Yokogawa microscope. Samples were then processed exactly as for TEM.

Live-CLEM. INS1 cells were cultured on gridded coverslips (MatTek P35G-1.5-14C-GRID). Live-cell imaging was performed using Inverted Nikon Eclipse Ti microscope (Nikon Ti PFS Spinning Disk) equipped with x100 TIRF objective (NA $=1.49$ ), with the PFS (perfect focus system), an EMCCD camera (Evolve, Photometrics) and Yokogawa CSU-X1 Confocal Scanner. GFP and m-Cherry were excited with a $488-\mathrm{nm}$ and a $561-\mathrm{nm}$ laser, respectively $(100 \mathrm{~mW})$. Live INS1 cells were imaged at 20 frames per second. 4D imaging was performed using consecutive $\mathrm{xy}(\mathrm{c}) \mathrm{tz}$ time-lapse acquisitions. Time series of $2 \mathrm{D}$ sections were analyzed using FIJI and IMARIS software. In the end of experiment, the cells were immediately fixed in PFA 2.5\%, Glutaraldehyde $2.5 \%$ in sodium cacodylate $0.1 \mathrm{M}$ for $20 \mathrm{~min}$ at room temperature. The samples were then post fixed in $1 \%$ osmium tetroxide in $0.1 \mathrm{M}$ cacodylate buffer for $1 \mathrm{~h}$ at $4{ }^{\circ} \mathrm{C}$, dehydrated through graded alcohol $(50,70,90$, and $100 \%$ ), embedded in Epon 812 cut at $70 \mathrm{~nm}$ (Leica Ultracut UCT) and contrasted with uranyl acetate and lead citrate. Serial sections were examined using a Philips CM12 transmission electron microscope (CM12, Philips; FEI Electron Optics, Eindhoven, the Netherlands) operated at $80 \mathrm{kV}$ and equipped with an Orius 1000 CCD camera (Gatan, Pleasanton, USA).

On-section CLEM. High accuracy on-section CLEM was performed as previously described ${ }^{95,96}$, with minor modifications. Cells grown on sapphire disks (Engineering Office M. Wohlwend GmbH, Sennwald, Switzerland) were high pressure frozen with a BAL-TEC HPM 010 machine. Freeze substitution (FS) and resin infiltration were performed in a Leica AFS2 machine. Cells monolayers were freeze substituted with $0.1 \%$ uranyl acetate in acetone for $9 \mathrm{~h}$ at $-90^{\circ} \mathrm{C}$. The temperature was then increased $\left(5^{\circ} \mathrm{C} / \mathrm{h}\right)$ to $-45^{\circ} \mathrm{C}$ and samples were incubated for $5 \mathrm{~h}$. The FS solution was then washed out with dry acetone and samples were infiltrated with Lowicryl HM20 (Polyscience Europe GmbH, Eppelheim, Germany) at increasing concentration $(10 \%, 25 \%, 50 \%, 75 \%, 3 \times 100 \%)$, while the temperature was increased to $-25^{\circ} \mathrm{C}$. The resin was then polymerized under UV light for $48 \mathrm{~h}$. In all, 300-nm-thick sections were cut from the polymerized resin block and picked up on carbon coated mesh grids. TetraSpeck fluorescent microspheres $(100 \mathrm{~nm})$ (Lifetechnologies) were adsorbed to the grid for the subsequent fiducial-based correlation between light (LM) and electron microscopy (EM) images. Fluorescence imaging of the sections was performed as in Kukulski et al..$^{95}$ using an Olympus ScanR microscope and a $100 \times 1.4$ NA oil objective. Tilt series of the areas with fluorescent structures of interest were acquired with a FEI Tecnai F30 electron microscope. Tomograms were then reconstructed using the IMOD software package $^{97}$. Correlation of LM and EM images was performed by manually assigning the position of tetraspecks in the two imaging modalities using ec-CLEM plugin $^{98}$ from ICY software ${ }^{99}$.

FIB-SEM. Freshly isolated pancreatic islets were fixed with $2.5 \%$ Glutaraldehyde (Electron Microscopy Sciences), 0.1\% Malachite green oxalate (Sigma) in $0.1 \mathrm{M}$ PHEM buffer. Subsequently, islets were washed with $0.1 \mathrm{M}$ PHEM buffer and stored at $4{ }^{\circ} \mathrm{C}$ until further processing in a PELCO BioWave Pro microwave processor (Ted Pella, Inc.) ${ }^{100}$. The processing procedure was as follows: postfixation with $1 \%$ osmium tetroxide $\left(\mathrm{OsO}_{4}\right.$; Electron Microscopy Sciences) $+0.8 \%$ potassium hexacyanoferrate (III) $\left(\mathrm{K}_{3} \mathrm{Fe}(\mathrm{CN})_{6}\right.$; Merck) in $\mathrm{H}_{2} \mathrm{O}$, rinsed with $\mathrm{H}_{2} \mathrm{O}$, post-stained with $1 \%$ tannic acid, rinsed with $\mathrm{H}_{2} \mathrm{O}$, en bloc stained with $0.5 \%$ UA in $\mathrm{H}_{2} \mathrm{O}$, rinsed with $\mathrm{H}_{2} \mathrm{O}$, dehydrated with increasing concentrations of ethanol $(25,50,75,90$, and $100 \%)$ and embedded with a graded series of Durcupan (SigmaAldrich). Subsequently the Durcupan infiltrated islets were incubated at $60^{\circ} \mathrm{C}$ for $48 \mathrm{~h}$ to polymerize the resin. Once polymerized, resin embedded islets were trimmed to expose two surfaces of material. After trimming, the samples were mounted onto the edge of an SEM stub (Agar Scientific) with silver conductive epoxy (CircuitWorks) with one of the trimmed surfaces facing up so that it was perpendicular to the focused ion beam (FIB). The sample was then sputter coated with gold ( $180 \mathrm{~s}$ at $30 \mathrm{~mA})$ in a Quorum Q150RS coater before being placed in the Zeiss Auriga 60 focused ion beam scanning electron microscope (FIB-SEM). Once the ROI was located in the sample, Atlas3D software (Fibics Inc. and Zeiss) was used to perform sample preparation and 3D acquisitions. First a platinum protective coat of $(20 \mu \mathrm{m} \times 20 \mu \mathrm{m})$ was deposited with $1 \mathrm{nA}$ FIB current. A small rough trench was then milled to expose the imaging cross-section with $16 \mathrm{nA}$ FIB current, followed with a polish at $4 \mathrm{nA}$. The 3D acquisition milling was done with $1 \mathrm{nA}$ FIB current. For SEM imaging, the beam was operated at $1.5 \mathrm{kV}$ with $60 \mu \mathrm{m}$ aperture using High Current mode and the EsB detector $(1.1 \mathrm{kV}$ collector voltage $)$ at a dwell time from $10 \mu \mathrm{s}$ with no line averaging over a pixel size of $4 \times 4 \mathrm{~nm}$ and slice thickness $8 \mathrm{~nm}$. All acquired images were then combined as a stack with FIJI (ImageJ) and subsequently aligned using the TrakEM2 plugin. Finally, the contrast of all images was inverted to have the same contrast as that of conventional TEM images.

Generation of Compound A selectively inhibiting MAPK p38d. Following the screening of a set of 808000 compounds from the Sanofi proprietary compound collection using a HTRF assay on purified p $38 \delta$ with ATF2 as substrate, compounds of the pyrimido-azepinone family were identified as moderate inhibitors of p38 . After a short chemical optimization cycle, Compound A (Supplementary Fig. 11a) was selected as a tool compound to study the effects of $\mathrm{p} 38 \delta$ inhibition in various in vitro assays and on blood glucose levels in BTBR $o b / o b$ mice described in this paper.

Compound A demonstrated a very good selectivity profile versus $\mathrm{p} 38 \alpha$ and PKD1, as measured by mobility shift assays (Caliper) while it showed an equivalent inhibitory effect against p38 $\gamma$ (Supplementary Fig. 11b). Furthermore, Compound A exhibited a very good selectivity profile in a panel of 60 kinases (Supplementary Fig. 11c) with the exception of p70S6K (59\% inhibition at $10 \mu \mathrm{M})$ and mTOR $(48 \%$ inhibition at $10 \mu \mathrm{M}$ ). Inhibition of $\mathrm{p} 38 \gamma$ was also confirmed in this panel (alias SAPK3: $64 \%$ inhibition at $10 \mu \mathrm{M}$ ). A more detailed account of the discovery, chemistry and biochemical properties of this novel class of selective p $38 \delta$ inhibitors will be given elsewhere.

Statistical analysis. Sample sizes for all types of experiments in this study were chosen according to published guidelines and to our previous experience. In all imaging-based quantitative experiments, systematic uniform random sampling was used. The statistical significance of the differences between two groups was investigated by $t$-test or by Mann-Whitney $U$-test. At least three independent experiments were performed for each comparison. The statistical analysis was performed using Excel or GraphPad Prism software (GraphPad Software Inc.).

Reporting summary. Further information on research design is available in the Nature Research Reporting Summary linked to this article.

\section{Data availability}

All relevant data that support the conclusions of this study are available from the authors on reasonable request (see author contributions for specific data sets). The source data underlying Figs. 1b, c, e, 2b, c, e, 3b, e, 4a-c, e, i, j, and 5b, f and Supplementary Figs. $3 \mathrm{a}-\mathrm{c}, 4 \mathrm{a}, \mathrm{b}, \mathrm{g}, \mathrm{h}, 6,7 \mathrm{a}-\mathrm{c}, 8 \mathrm{a}, \mathrm{b}, 9,10,11 \mathrm{~d}, 12 \mathrm{a}, \mathrm{c}, \mathrm{d}$, and $13 \mathrm{a}-\mathrm{c}$ are provided as a Source Data file. Uncropped immunoblots are shown in Supplementary Fig. 14. Analysis of PKD1 (Prkcm) expression in islets of wt and ob/ob BTBR mice ${ }^{45}$ has been performed at http://diabetes.wisc.edu/

Received: 26 August 2017 Accepted: 27 June 2019

Published online: 25 July 2019

\section{References}

1. Ohsumi, Y. Historical landmarks of autophagy research. Cell Res. 24, 9-23 (2013).

2. Efeyan, A., Comb, W. \& Sabatini, D. Nutrient-sensing mechanisms and pathways. Nature 517, 302-310 (2015).

3. Kaur, J. \& Debnath, J. Autophagy at the crossroads of catabolism and anabolism. Nat. Rev. Mol. Cell Biol. 16, 461-472 (2015).

4. Lamb, C., Yoshimori, T. \& Tooze, S. The autophagosome: origins unknown, biogenesis complex. Nat. Rev. Mol. Cell Biol. 14, 759-774 (2013).

5. Ebato, C. et al. Autophagy is important in islet homeostasis and compensatory increase of beta cell mass in response to high-fat diet. Cell Metab. 8, 325-332 (2008).

6. Chu, K., O’Reilly, L., Ramm, G. \& Biden, T. J. High-fat diet increases autophagic flux in pancreatic beta cells in vivo and ex vivo in mice. Diabetologia 58, 2074-2078 (2015).

7. Quan, W. et al. Autophagy deficiency in beta cells leads to compromised unfolded protein response and progression from obesity to diabetes in mice. Diabetologia 55, 392-403 (2012).

8. Bachar-Wikstrom, E., Wikstrom, J., Kaiser, N., Cerasi, E. \& Leibowitz, G. Improvement of ER stress-induced diabetes by stimulating autophagy. Autophagy 9, 626-628 (2013). 
9. Rivera, J. et al. Human-IAPP disrupts the autophagy/lysosomal pathway in pancreatic $\beta$-cells: protective role of p62-positive cytoplasmic inclusions. Cell Death Differ. 18, cdd2010111 (2010).

10. Shigihara, N. et al. Human IAPP-induced pancreatic $\beta$ cell toxicity and its regulation by autophagy. J. Clin. Invest. 124, 3634-3644 (2014).

11. Rivera, J., Costes, S., Gurlo, T., Glabe, C. \& Butler, P. Autophagy defends pancreatic $\beta$ cells from human islet amyloid polypeptide-induced toxicity. J. Clin. Invest. 124, 3489-3500 (2014).

12. Kim, J. et al. Amyloidogenic peptide oligomer accumulation in autophagydeficient $\beta$ cells induces diabetes. J. Clin. Invest. 124, 3311-3324 (2014).

13. Las, G., Serada, S. B., Wikstrom, J. D., Twig, G. \& Shirihai, O. S. Fatty acids suppress autophagic turnover in $\beta$-cells. J. Biol. Chem. 286, 42534-42544 (2011).

14. Martino, L. et al. Palmitate activates autophagy in INS-1E $\beta$-cells and in isolated rat and human pancreatic islets. PLoS One 7, e36188 (2012).

15. Cnop, M. et al. RNA sequencing identifies dysregulation of the human pancreatic islet transcriptome by the saturated fatty acid palmitate. Diabetes 63, 1978-1993 (2014).

16. Mir, S. U. et al. Inhibition of autophagic turnover in $\beta$-cells by fatty acids and glucose leads to apoptotic cell death. J. Biol. Chem. 290, 6071-6085 (2015).

17. Komiya, K. et al. Free fatty acids stimulate autophagy in pancreatic $\beta$-cells via JNK pathway. Biochem. Biophys. Res. Commun. 401, 561-567 (2010).

18. Zummo, F. P. et al. Glucagon-like peptide-1 protects pancreatic beta-cells from death by increasing autophagic flux and restoring lysosomal function. Diabetes 66, 1272-1285 (2017).

19. Janikiewicz, J. et al. Inhibition of SCD1 impairs palmitate-derived autophagy at the step of autophagosome-lysosome fusion in pancreatic $\beta$-cells. J. Lipid Res. 56, 1901-1911 (2015).

20. Masini, M. et al. Autophagy in human type 2 diabetes pancreatic beta cells. Diabetologia 52, 1083-1086 (2009).

21. Masini, M. et al. Ultrastructural alterations of pancreatic beta cells in human diabetes mellitus. Diabetes Metab. Res. Rev. 33, e2894 (2017).

22. Schuit, F., PAI, V. \& Pipeleers, D. Glucose stimulates proinsulin biosynthesis by a dose-dependent recruitment of pancreatic beta cells. Proc. Natl Acad. Sci. USA 85, 3865-3869 (1988).

23. Prentki, M., Matschinsky, F. \& Madiraju, S. Metabolic signaling in fuelinduced insulin secretion. Cell Metab. 18, 162-185 (2013).

24. Weir, G. Islet-cell biology in 2015: understanding secretion, ageing and death in $\beta$ cells. Nat. Rev. Endocrinol. 12, 72-74 (2016).

25. Meda, P. Lysosomes in normal pancreatic beta cells. Diabetologia 14, 305-310 (1978).

26. Orci, L. et al. Insulin, not C-peptide (proinsulin), is present in crinophagic bodies of the pancreatic B-cell. J. Cell Biol. 98, 222-228 (1984).

27. Uchizono, Y., Alarcón, C., Wicksteed, B., Marsh, B. \& Rhodes, C. The balance between proinsulin biosynthesis and insulin secretion: where can imbalance lead? Diabetes Obes. Metab. 9, 56-66 (2007).

28. Marsh, B. et al. Regulated autophagy controls hormone content in secretorydeficient pancreatic endocrine beta-cells. Mol. Endocrinol. 21, 2255-2269 (2007).

29. Zhang, X. et al. Substrate-favored lysosomal and proteasomal pathways participate in the normal balance control of insulin precursor maturation and disposal in $\beta$-cells. PLoS ONE 6, e27647 (2011).

30. Riahi, Y. et al. Autophagy is a major regulator of beta cell insulin homeostasis. Diabetologia 59, 1480-1491 (2015).

31. Yamaguchi, H. et al. Golgi membrane-associated degradation pathway in yeast and mammals. EMBO J. 35, 1991-2007 (2016).

32. Goginashvili, A. et al. Insulin granules. Insulin secretory granules control autophagy in pancreatic $\beta$ cells. Science 347, 878-882 (2015).

33. Poitout, V. et al. Glucolipotoxicity of the pancreatic beta cell. Biochim. Biophys. Acta 1801, 289-298 (2010).

34. Ran, F. et al. Genome engineering using the CRISPR-Cas9 system. Nat. Protoc. 8, 2281-2308 (2013).

35. Sharlow, E. et al. Potent and selective disruption of protein kinase D functionality by a benzoxoloazepinolone. J. Biol. Chem. 283, 33516-33526 (2008).

36. Tanida, I., Ueno, T. \& Kominami, E. LC3 conjugation system in mammalian autophagy. Int. J. Biochem. Cell Biol. 36, 2503-2518 (2004).

37. Yamamoto, A. et al. Bafilomycin Al prevents maturation of autophagic vacuoles by inhibiting fusion between autophagosomes and lysosomes in rat hepatoma cell line, H-4-II-E cells. Cell Struct. Funct. 23, 33-42 (1998).

38. Mauvezin, C., Nagy, P., Juh'asz, G. \& Neufeld, T. Autophagosome-lysosome fusion is independent of V-ATPase-mediated acidification. Nat. Commun. $\mathbf{6}$, 7007 (2015).

39. Sancak, Y. et al. The Rag GTPases bind raptor and mediate amino acid signaling to mTORC1. Science 320, 1496-1501 (2008).

40. Sancak, Y. et al. Ragulator-rag complex targets mTORC1 to the lysosomal surface and is necessary for its activation by amino acids. Cell 141, 290-303 (2010).
41. Zoncu, R. et al. mTORC1 senses lysosomal amino acids through an inside-out mechanism that requires the vacuolar $\mathrm{H}(+)$-ATPase. Science 334, 678-683 (2011).

42. Clee, S. M., Nadler, S. T. \& Attie, A. D. Genetic and Genomic Studies of the BTBR ob/ob mouse model of type 2 diabetes. Am. J. Ther. 12, 491 (2005).

43. Bjørkøy, G. et al. p62/SQSTM1 forms protein aggregates degraded by autophagy and has a protective effect on huntingtin-induced cell death. J. Cell Biol. 171, 603-614 (2005)

44. Jung, $H$. et al. Loss of autophagy diminishes pancreatic $\beta$ cell mass and function with resultant hyperglycemia. Cell Metab. 8, 318-324 (2008).

45. Keller, M. P. et al. A gene expression network model of type 2 diabetes links cell cycle regulation in islets with diabetes susceptibility. Genome Res. 18, 706-716 (2008)

46. Sumara, G. et al. Regulation of PKD by the MAPKp38ס in insulin secretion and glucose homeostasis. Cell 136, 235-248 (2009).

47. Kebede, M. A. et al. SORCS1 is necessary for normal insulin secretory granule biogenesis in metabolically stressed $\beta$ cells. J. Clin. Invest. 124, 4240-4256 (2014).

48. Gamp, A.-C. et al. LIMP-2/LGP85 deficiency causes ureteric pelvic junction obstruction, deafness and peripheral neuropathy in mice. Hum. Mol. Genet. 12, 631-646 (2003).

49. Tanaka, Y. et al. Accumulation of autophagic vacuoles and cardiomyopathy in LAMP-2-deficient mice. Nature 406, 902 (2000).

50. Schröder, J. et al. Deficiency of the tetraspanin CD63 associated with kidney pathology but normal lysosomal function. Mol. Cell Biol. 29, 1083-1094 (2008).

51. Lee, M. Role of islet $\beta$ cell autophagy in the pathogenesis of diabetes. Trends Endocrinol. Metab. 25, 620-627 (2014).

52. Bartolomé, A. et al. Pancreatic $\beta$-cell failure mediated by mTORC1 hyperactivity and autophagic impairment. Diabetes 63, 2996-3008 (2014).

53. Osório, J. Diabetes: protective role of autophagy in pancreatic $\beta$ cells. Nat. Rev Endocrinol. 10, 575-575 (2014)

54. Watada, H. \& Fujitani, Y. Minireview: autophagy in pancreatic $\beta$-cells and its implication in diabetes. Mol. Endocrinol. 29, 338-348 (2015).

55. Olson, L., Redmon, J., Towle, H. \& Robertson, R. Chronic exposure of HIT cells to high glucose concentrations paradoxically decreases insulin gene transcription and alters binding of insulin gene regulatory protein. J. Clin. Invest. 92, 514-519 (1993).

56. Poitout, V. et al. Regulation of the insulin gene by glucose and fatty acids. J. Nutr. 136, 873-876 (2006).

57. Welsh, M., Nielsen, D., MacKrell, A. \& Steiner, D. Control of insulin gene expression in pancreatic beta-cells and in an insulin-producing cell line, RIN5F cells. II. Regulation of insulin mRNA stability. J. Biol. Chem. 260, 13590-13594 (1985).

58. Knoch, K.-P. et al. Polypyrimidine tract-binding protein promotes insulin secretory granule biogenesis. Nat. Cell Biol. 6, 207-214 (2004).

59. Ehehalt, F. et al. Impaired insulin turnover in islets from type 2 diabetic patients. Islets 2, 30-36 (2010).

60. Sun, J. et al. Proinsulin misfolding and endoplasmic reticulum stress during the development and progression of diabetes. Mol. Aspects Med. 42, 105-118 (2015).

61. Alarcon, C. et al. Pancreatic $\beta$-cell adaptive plasticity in obesity increases insulin production but adversely affects secretory function. Diabetes $\mathbf{6 5}$, 438-450 (2016).

62. Kahn, S. E. \& Halban, P. A. Release of incompletely processed proinsulin is the cause of the disproportionate proinsulinemia of NIDDM. Diabetes 46, 1725-1732 (1997).

63. Boland, B. B., Rhodes, C. J. \& Grimsby, J. S. The dynamic plasticity of insulin production in $\beta$-cells. Mol. Metab. 6, 958-973 (2017).

64. Ward, W. et al. Disproportionate elevation of immunoreactive proinsulin in Type 2 (non-insulin-dependent) diabetes mellitus and in experimental insulin resistance. Diabetologia 30, 698-702 (1987).

65. Røder, M., Porte, D., Schwartz, R. \& Kahn, S. Disproportionately elevated proinsulin levels reflect the degree of impaired B cell secretory capacity in patients with noninsulin-dependent diabetes mellitus. J. Clin. Endocrinol. Metab. 83, 604-608 (1998).

66. Gonzalez-Duque, S. et al. Conventional and neo-antigenic peptides presented by $\beta$ cells are targeted by circulating naïve $\mathrm{CD} 8+\mathrm{T}$ cells in type 1 diabetic and healthy donors. Cell Metab. https://doi.org/10.1016/..cmet.2018.07.007 (2018).

67. Wan, X. et al. Pancreatic islets communicate with lymphoid tissues via exocytosis of insulin peptides. Nature 560, 107-111 (2018).

68. Kim, Y.-M. et al. mTORC1 phosphorylates UVRAG to negatively regulate autophagosome and endosome maturation. Mol. Cell 57, 207-218 (2015).

69. Cheng, X. et al. Pacer is a mediator of mTORC1 and GSK3-TIP60 signaling in regulation of autophagosome maturation and lipid metabolism. Mol Cell. https://doi.org/10.1016/j.molcel.2018.12.017 (2019). 
70. Fraenkel, M. et al. mTOR inhibition by rapamycin prevents $\beta$-cell adaptation to hyperglycemia and exacerbates the metabolic state in type 2 diabetes. Diabetes 57, 945-957 (2008).

71. Blandino-Rosano, M. et al. Loss of mTORC1 signalling impairs $\beta$-cell homeostasis and insulin processing. Nat. Commun. 8, 16014 (2017).

72. Mészáros, G., Pasquier, A., Vivot, K., Goginashvili, A. \& Ricci, R. Lysosomes in nutrient signalling: a focus on pancreatic $\beta$-cells. Diabetes Obes. Metab. 20, 104-115 (2018).

73. Brandhorst, S. et al. A periodic diet that mimics fasting promotes multi-system regeneration, enhanced cognitive performance, and healthspan. Cell Metab. 22, 86-99 (2015)

74. Samuel, V. T. \& Shulman, G. I. Mechanisms for insulin resistance: common threads and missing links. Cell 148, 852-871 (2012).

75. Lund, A., Bagger, J., Christensen, M., Knop, F. \& Vilsboll, T. Glucagon and type 2 diabetes: the return of the alpha cell. Curr. Diabetes Rep. 14, 84 (2014).

76. Kong, K. et al. M3-muscarinic receptor promotes insulin release via receptor phosphorylation/arrestin-dependent activation of protein kinase D1. Proc. Natl Acad. Sci. USA 107, 21181-21186 (2010).

77. Gehart, H. et al. The BAR domain protein arfaptin-1 controls secretory granule biogenesis at the trans-golgi network. Dev. Cell 23, 756-768 (2012)

78. Ferdaoussi, M. et al. G protein-coupled receptor (GPR)40-dependent potentiation of insulin secretion in mouse islets is mediated by protein kinase D1. Diabetologia 55, 2682-2692 (2012)

79. Bergeron, V. et al. Deletion of protein kinase D1 in pancreatic beta cells impairs insulin secretion in high-fat fed mice. Diabetes 67, 71-77 (2017).

80. Speliotes, E. et al. Association analyses of 249,796 individuals reveal 18 new loci associated with body mass index. Nat. Genet. 42, 937-948 (2010).

81. Graff, M. et al. Genome-wide analysis of BMI in adolescents and young adults reveals additional insight into the effects of genetic loci over the life course. Hum. Mol. Genet. 22, 3597-3607 (2013).

82. Locke, A. et al. Genetic studies of body mass index yield new insights for obesity biology. Nature 518, 197-206 (2015).

83. Hsa, S. et al. A genome-wide search for type 2 diabetes susceptibility genes in an extended Arab family. Ann. Hum. Genet. 77, 488-503 (2013).

84. Takino, T. et al. Tetraspanin CD63 promotes targeting and lysosomal proteolysis of membrane-type 1 matrix metalloproteinase. Biochem. Biophys. Res. Commun. 304, 160-166 (2003).

85. Van Niel, G. et al. The tetraspanin CD63 regulates ESCRT-independent and -dependent endosomal sorting during melanogenesis. Dev. Cell 21, 708-721 (2011).

86. Pols, M.S. \& Klumperman, J. Trafficking and function of the tetraspanin CD63. Exp. Cell Res. 315, 1584-1592 (2009).

87. Smith, R. E. \& Farquhar, M. G. Lysosome function in the regulation of the secretory process in cells of the anterior pituitary gland. J. Cell Biol. 31 319-347 (1966).

88. Orci, L., Junod, A., Pictet, R., Renold, A. \& Rouiller, C. Granulolysis in A-cells of endocrine pancreas in spontaneous and experimental diabetes in animals. $J$. Cell Biol. 38, 462-466 (1968).

89. Landström, A. H., Andersson, A. \& Borg, L. A. H. Lysosomes and pancreatic islet function: adaptation of $\beta$-cell lysosomes to various metabolic demands. Metabolism 40, 399-405 (1991).

90. De Duve, C. The lysosome in retrospect. Ann. N. Y. Acad. Sci. 804, 1-10 (1996).

91. Csizmadia, T. et al. Molecular mechanisms of developmentally programmed crinophagy in Drosophila. J. Cell Biol. 217, 361-374 (2017).

92. Brereton, M., Rohm, M. \& Ashcroft, F. $\beta$-Cell dysfunction in diabetes: a crisis of identity? Diabetes Obes. Metab. 18, 102-109 (2016).

93. Gallegos, L. L., Kunkel, M. T. \& Newton, A. C. Targeting protein kinase C activity reporter to discrete intracellular regions reveals spatiotemporal differences in agonist-dependent signaling. J. Biol. Chem. 281, 30947-30956 (2006).

94. Lenormand, C. et al. Birbeck granule-like "organized smooth endoplasmic reticulum" resulting from the expression of a cytoplasmic YFP-tagged langerin. PLoS One 8, e60813 (2013).

95. Kukulski, W. et al. Methods in cell biology. Methods Cell Biol. 111, 235-257 (2012).

96. Avinoam, O., Schorb, M., Beese, C., Briggs, J. A. G. \& Kaksonen, M. Endocytic sites mature by continuous bending and remodeling of the clathrin coat. Science 348, 1369-1372 (2015).

97. Kremer, J., Mastronarde, D. \& McIntosh, J. Computer visualization of threedimensional image data using IMOD. J. Struct. Biol. 116, 71-76 (1996).

98. Paul-Gilloteaux, P. et al. eC-CLEM: flexible multidimensional registration software for correlative microscopies. Nat. Methods 14, 102-103 (2017).
99. Chaumont, F. et al. Icy: an open bioimage informatics platform for extended reproducible research. Nat. Methods 9, 690-696 (2012).

100. Karreman, M. et al. Correlating intravital multi-photon microscopy to $3 \mathrm{D}$ electron microscopy of invading tumor cells using anatomical reference points. PLoS ONE 9, el14448 (2014).

\section{Acknowledgements}

We thank all the facilities at the Institut de Génétique et de Biologie Moléculaire et Cellulaire for helping us with related experiments. We thank Izabela Sumara, all the current members of the Ricci laboratory, Valentina Lionello, Ksenia Krupina, Joëlle Morvan, and Helmuth Gehart for critical scientific input. This work was supported by the Agence Nationale de la Recherche (ANR) (AAPG 2017 LYSODIABETES), by the European Foundation for the Study of Diabetes (EFSD)/Lilly European Diabetes Research Programme grant, by a European Research Council (ERC) starting grant (ERC2011-StG, 281271-STRESS METABOL) and by the ANR-10-LABX-0030-INRT grant, a French State fund managed by the ANR under the frame program Investissements d'Avenir ANR-10-IDEX-0002-02. A. Pasquier was supported by a FRM fellowship (fin de thèse). A. Goginashvili and V. Aubert were supported by the ERC grant and the EFSD/ Lilly European Diabetes Research Programme grant. Z.Z. was supported by an EFSD/ Chinese Diabetes Society/Lilly Research fellowship. K.V. was supported by a FRM fellowship ARF20160936008 and an Individual Fellowship (798961 INSULYSOSOME) in the framework of the Marie-Sklodovska Curie actions of the European Commission. P.S. was supported by the DFG graduate school (GRK1459) and the DFG research unit (FOR2625). J.K-C.and F.P. were supported by the the Agence Nationale de la Recherche (ANR) (AAPG 2017 LYSODIABETES).

\section{Author contributions}

A.P., A.Goginashvili, and R.R. designed experiments and wrote the manuscript with input from all authors. A.P. performed experiments presented in Figs. 1a-e, 2b-e, 3a-f, 4a-j, and 5a-e, Supplementary Figs. 1-8 and 11-13. A.Goginashvili performed experiments presented in Figs. 1b, 2a, 3b, c, and 5a-d, Supplementary Figs. 1, 12, and 13. K.V. performed experiments presented in Figs. 4e and 5f and Supplementary Figs. 7-9 and 12 E.E. assisted in all immunofluorescence experiments, helped with the immunoblot presented in Fig. $2 \mathrm{~d}$ and with generation of INS1 ${ }^{\text {PGCD }}$ cells. C.S. prepared samples for TEM and CLEM, helped set-up the CLEM experiments, and with the acquisition and analysis of images in Fig. 1d-e, Supplementary Figs. 5a, 7c, and 13a. E.M., M.P., J.K.-C., and F.P. provided human islets. Z.Z. and V.A. helped generating INS1 ${ }^{\text {PGCD }}$ cells. Z.L. performed live-CLEM experiment shown in Supplementary Fig. 5b. M.S. helped with the characterization of CD63 expression. G.M. and A.Ganzhorn generated Compound A (Supplementary Fig. 10a-c). P.R., N.L.S., and Y.S. performed FIB-SEM and on-section CLEM presented in Fig. 3c, d (FIB-SEM) and Supplementary Fig. 2 (on-section CLEM). P.S. provided CD63 knockout mice and the antibody against CD63.

\section{Additional information}

Supplementary Information accompanies this paper at https://doi.org/10.1038/s41467019-11170-4.

Competing interests: The authors declare no competing interests.

Reprints and permission information is available online at http://npg.nature.com/ reprintsandpermissions/

Peer review information: Nature Communications thanks Myung-Shik Lee and other anonymous reviewer(s) for their contribution to the peer review of this work.

Publisher's note: Springer Nature remains neutral with regard to jurisdictional claims in published maps and institutional affiliations.

Open Access This article is licensed under a Creative Commons Attribution 4.0 International License, which permits use, sharing, adaptation, distribution and reproduction in any medium or format, as long as you give appropriate credit to the original author(s) and the source, provide a link to the Creative Commons license, and indicate if changes were made. The images or other third party material in this article are included in the article's Creative Commons license, unless indicated otherwise in a credit line to the material. If material is not included in the article's Creative Commons license and your intended use is not permitted by statutory regulation or exceeds the permitted use, you will need to obtain permission directly from the copyright holder. To view a copy of this license, visit http://creativecommons.org/ licenses/by/4.0/

(๑) The Author(s) 2019 